\title{
Indirect Estimation of Selected Measures of Fertility and Marital Fertility from Information on CWR (0-9): An Application to India /States /Districts
}

\author{
Krishna Murthy Ponnapalli ${ }^{1 *}$ and Ravi Kant Soren ${ }^{2}$ \\ ${ }^{1}$ Institute of Population Studies (IPoSt), Mekelle University, P.O.Box 231, Mekelle, Ethiopia \\ (*pkmurthy2001@yahoo.com). \\ ${ }^{2}$ International Institute for Population Studies (IIPS), Deonar, Mumbai-400 088, India.
}

\begin{abstract}
Fertility is one of the important components of population change, the other two being mortality and migration. When vital statistical data on number of births is readily available it is estimated directly using various direct measures of fertility such as the crude birth rate (CBR). When the information on number of births is not directly available, fertility is measured using the census information on child woman ratios (CWR) of various types. CWR has several limitations, but gained importance with the attempts made by Rele in 1963, and followed by several other researchers such as Hauer and his co-authors in 2013 deriving TFR from it. In the present study yet another attempt is made to use CWRs to derive not only TFR but also other summary indicators such as the TMFR. A set of simple mathematical formula have been used to estimate the fertility and marital fertility using the CWR of the ages $0-9$. The 2011 census age-sex data of districts in India was used to derive a set of fertility estimates for total, rural and urban areas of all districts. Further, these estimates of 2011 have been compared with the estimates of 2011 earlier made by Guilmoto and Rajan to analyse the robustness of the estimates.
\end{abstract}

Keywords: Fertility, Child Women Ratio, Indirect Estimation, Age-sex data, TFR, TMFR, India.

\section{INTRODUCTION}

The commonly used measures such as rates, ratios, percentages and probabilities in mathematics and statistics also play a great role in understanding various demographic phenomenon's such as fertility. Fertility is one of the important components of population change, the other two being mortality and migration. When vital statistical data on number of births is readily available it is estimated directly using various direct measures of fertility such as the crude birth rate (CBR). When the information on number of births not directly available fertility is measured using the census information on child woman ratios (CWR) of various types. CWR has its own merits and demerits. When using the child woman ratio (Children 0-4/ Women 15-49) as an indicator of fertility we assume that children enumerated by the time of the census in the age group 0-4 are the actual number of children born 5 years ago and those surviving. The logic behind is that it is assumed that mortality is negligible on the Children 0-4 and the Women 15-49, and also assume that no migration during the last five years of the census enumeration date; then the children 0-4 
enumerated at the census date equals to those born 5 years ago and surviving till today and thus, nothing but the number of births taken place during the past five years. When a child women ratio is constructed using this data then the CWR0-4 refers to 2.5 years before the census date as $\mathrm{C} 0-4$ here refers to the average number of births during the five year period before the census. If there is considerable amount of the impact of mortality and migration on the child women ratio, one has to adjust the measure for the above. Experience shows that one may use the child women ratio as a rough indicator of fertility without much problem, assuming a negligible impact of mortality and migration.

Rele $(1976,1987)$ suggested use of various types of child woman ratios (CWRs) to estimate more sophisticated indicators such as TFR and GRR indirectly. Recently, Hauer et al. (2013) suggested another simple method to estimate TFR from the information on CWR. When the infant mortality is comparatively insignificant, and also no migration among the women and dependent children the method suggested by them is observed to give plausible estimates of TFR. Smith (1992) suggested various measures of fertility simply by means of exploiting the interrelationships existing among different measures. Inspired by the above works namely by Hauer et al. (2013); Smith (1992); and Rele (1976, 1987), this paper makes an attempt to suggest a new methodology which does not require serious assumptions, not much input data but gives robust estimates of various measures of fertility even for sub-national populations in the absence of essential information on number of births but just by using the CWRs.

The data required for estimation of various measures of fertility and mortality is traditionally collected from three important sources namely: Censuses, Civil Registration System, Special surveys such as Sample Registration System (SRS) in India. All the above three sources have their own merits and demerits. Census and special surveys provide the information on children and women also and so following Rele (1976) one may compute a variety of child women ratios as per the need such as $\mathrm{CWR}_{0-4}(\mathrm{C} 0-4 / \mathrm{W} 15-49), \mathrm{CWR}_{5-9}(\mathrm{C} 5-9 / \mathrm{W} 20-54), \mathrm{CWR}_{0-9}(\mathrm{C} 0-9 / \mathrm{W} 15-54)$, where C stands for Children, W stands for Women. The advantage of this method is that it is possible to derive fertility measures even for the smallest area like village as CWR available at the local level.

Growing importance for decentralized planning in India and other countries over time made the governments and many a researcher to search for alternative ways of obtaining reliable estimates of fertility and mortality at below state level units such as districts, natural regions of a state, 
selected cities, etc. Recent special surveys of India namely National Family Health Survey (NFHS), District Level Household Survey (DLHS), Annual Health Survey (AHS) and others are best examples for such an alternative ways. Like the traditional sources, the above alternative sources of information also have their merits and demerits.

Indirect estimation of fertility and mortality using different alternative data sources, alternative methodologies and mixed methods/data sources thus started playing an important role in many of both developed and developing countries. A review of existing literature on indirect estimation of fertility and mortality carried out by several scholars in India and abroad clearly indicates that there is still a need for the development of new procedures and use of new data sources: (1) for a better understanding of the fertility and mortality processes at sub-state level units such as districts in India, (2) for a better evaluation of data gathered on fertility and mortality from various newly developed data sources such as SRS, NFHS, DLHS, etc., (3) for better assisting the various governments in their needs for the decentralized planning and policy implementation. However, most of the sophisticated indirect techniques suggested so far require stringent assumptions, huge amount of input data and thus make them not much applicable to derive estimates for below the state level such as the districts, towns, villages in India.

Further, to the knowledge of the present researchers (1) no researcher tried to make use of the marital status distribution data of India especially of the 2011 census in deriving indirectly the fertility measures like CBR, TFR, GMFR and TMFR (2) no attempt was made by any researcher to provide the above indirect estimates for the rural and urban areas of various districts. Thus the present study aims (1) to provide a new, simple and also innovative thought provoking approach for the derivation of various fertility measures/formulae which no one tried before; (2) to make use of the marital status distribution of age-sex data of 2011 census data to derive a new set of fertility indicators for districts in India. To be specific, the objectives of this study are (1) to develop a simple indirect technique for estimating CBR, TFR and TMFR and other related fertility measures from the information on the age-sex and marital status distribution of the population; (2) to derive plausible estimates of the above measures for districts/States/UTs in India using 2011 census age-sex marital status distribution data; and (3) to provide above estimates even for Rural and Urban areas of all districts in India.

In the following sections the paper presents the details of the data sources, methodological details and analysis of the results. 


\section{DATA AND METHODOLOGY}

\subsection{Data}

The data required for the present study on children of ages 0-9 years and women (or females) of ages 15 to 54 years, is collected from the marital status data by age-sex distribution of the 2011 census of the Registrar General of Indi (RGI). Soft copy of the 2011 census age-sex marital status distribution of data of various States/UTs/Districts in India was downloaded from the site http://www.censusindia.go.in. The quality of age-sex data of 2011 census seems to be good and has been improved over the period due to increasing literacy among women and a great exposure to mass media such as televisions, mobiles, etc.

\subsection{Methodology}

The methodology suggested in this study is explained as below:

In simple terms, to derive various fertility measures indirectly let us define at first that

C0-9 = Child population of ages 0 to 9

TP = Total population of both sexes

TFP15-54 = Total female population of the ages 15 to 54

MFP15-54 = Married female population of ages 15-54

CBR = Crude birth rate

GFR = General fertility rate

$T F R=$ Total fertility rate

GRR = Gross reproduction rate

$G M F R=$ General marital fertility rate

TMFR = Total marital fertility rate

Then, one can derive various fertility measures indirectly using the following formulae:

$C B R=((C 0-9 / 10) / T P) \times 1000$

$G F R=((C 0-9 / 10) / T F P 15-54) \times 1000$

$T F R=(G F R / 1000) \times 35$

GRR $=\operatorname{TFR} x(1 /(1+S R B))=(T F R / 2.05) ; S R B=$ Sex Ratio at Birth

GMFR $=((C 0-9 / 10) / M F P 15-54) \times 1000$

$T M F R=(G M F R / 1000) x 40$

The present methodology involved using various types of child/women or child/population ratios. The results obtained using the above formulae may be affected by some of the assumptions made by the researchers. 
In the above formulae of CBR, 10 represents the number of years and refer to the fact that children of ages 0 to 9 years were born 10 years before the census date. Following Hauer et al. (2013) dividing C0-9 by 10 leads to an indirect estimate of the number of births, assuming that the impact of mortality and migration is very negligible. Similar is the explanation in case of other measures. GFR is normally derived using TFP aged 15-49. Here in deriving GFR we considered TFP15-54, as its numerator consists of C0-9 and taking TFP15-54 is meaningful.

The formula given here for GRR calculation is generally considered as an indirect estimation procedure. This approach is mostly used by demographers as normally this formula also lead to a good estimate of GRR. In the GRR formulae, the factor 2.05 is obtained by considering 105 male births for 100 female births. When sex ratio at birth is available one may us the actual sex ratios at birth. But the above assumption of 1.05 SRB is acceptable in general.

Following Smith (1992) it is also suggested here to use the figures 35 and 40 in the formulae of TFR and TMFR respectively; and they are merely the assumed values of the reproductive life time of a typical woman of any developing country such as India. One may use in fact 35 in case of TMFR also (instead of 40) but on the safer side it is assumed here that the currently married women on an average may take some more time than the other in completing their fertility time period, especially in the developing countries of Asia and Africa.

All the fertility estimates provided here for districts in India were derived using the 2011 census data, however refer to the year 2006.5 or approximately the year 2007. Because as a matter of fact all the fertility estimates we derive using children 0-9 that refer to the births centered between 0-9 years before the census date.

It is assumed that the input data was not affected (or affected negligibly) by infant mortality and migration factors in the previous 10 years. This assumption we may make to be realized sometimes may not be applicable to areas such as Jammu and Kashmir where continuous disturbances are existing and the input data also affected. So, one should be cautious while interpreting the results for such areas as JK and its sub-regions.

\section{RESULTS AND DISCUSSION}

\subsection{Testing the Validity of the Estimates}

Validity of the derived estimates of CBR and TFR is made by comparing them with that of the indirect estimates of CBR and TFR earlier derived by Guilmoto and Rajan (2013). Guilmoto and 
Rajan (2013) used a modified version of the reverse survival method (MRSM) earlier suggested by Bhat (1996).

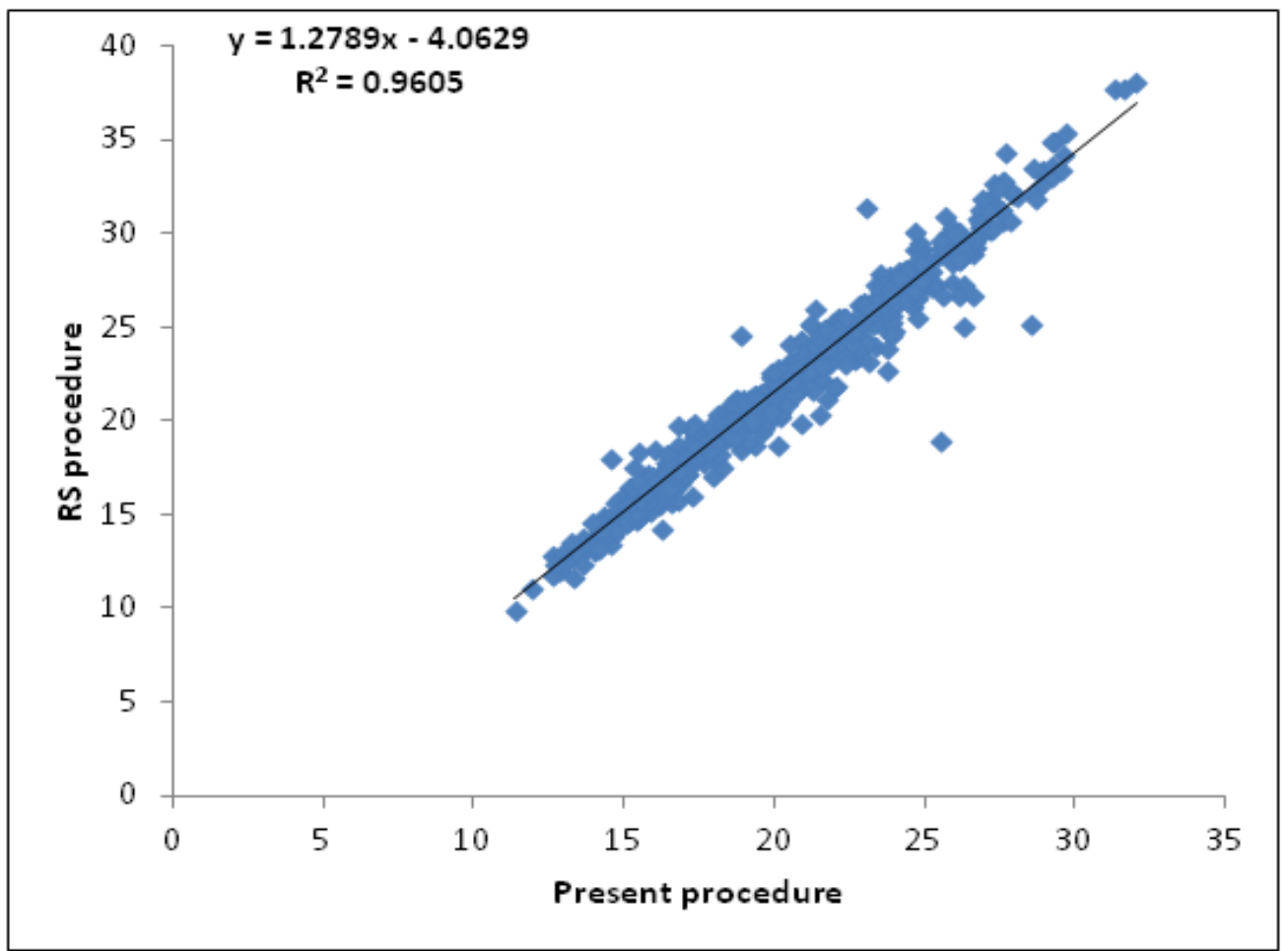

Figure 1. Comparison of estimates of CBR from MRSM with the present procedure.

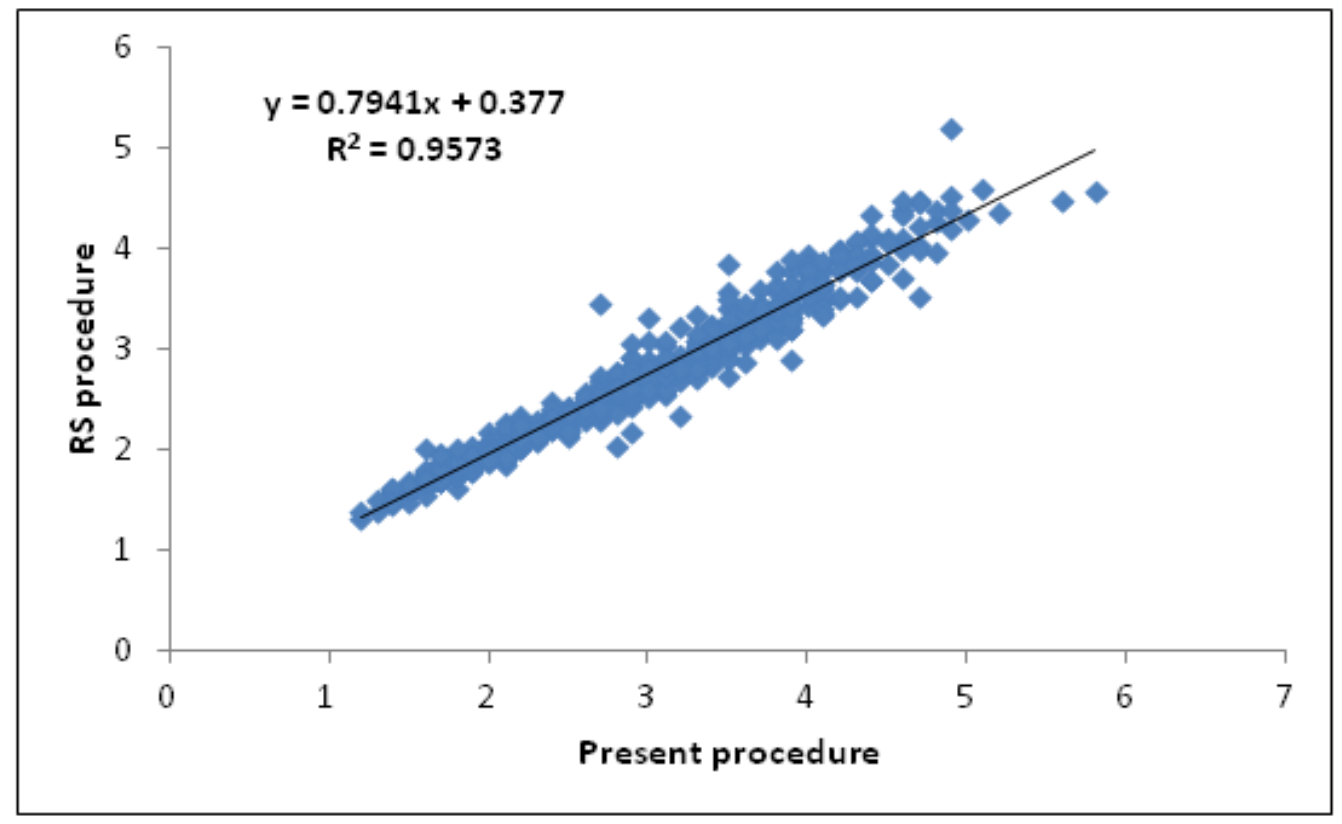

Figure 2. Comparison of the estimates of TFR from MRSM using the present procedure. 
Comparison of the regression lines fitted between TFR and CBR made by the two indirect estimation procedures (See Figs 1 and 2) clearly indicates that CBR and TFR estimates derived by using the present methodology are quite comparable. The percent variation explained by the simple linear regression model i.e., $\mathrm{R}^{2}$ is observed to be very high $(0.96)$ in both cases. Thus, other derived measures may also be considered acceptable for all practical purposes. Appendix (Table 1) provides CBR, TFR and TMFR estimates indirectly derived by the present methodology for the total, rural and urban of all India, States and Districts for the time period 2007 derived using the CWR(0-9) values of the 2011 census. TFR and CBR values used in the figures 1 and 2 are taken from the same table.

\subsection{Analysis of the Results}

For convenience state level estimates were provided graphically in Figures 3, 4 and 5 for the indicators of CBR (per 1000 population), TFR (per woman) and TMFR (per woman) for total, rural and urban areas. Results in the diagrams are as expected and are self-explanatory.

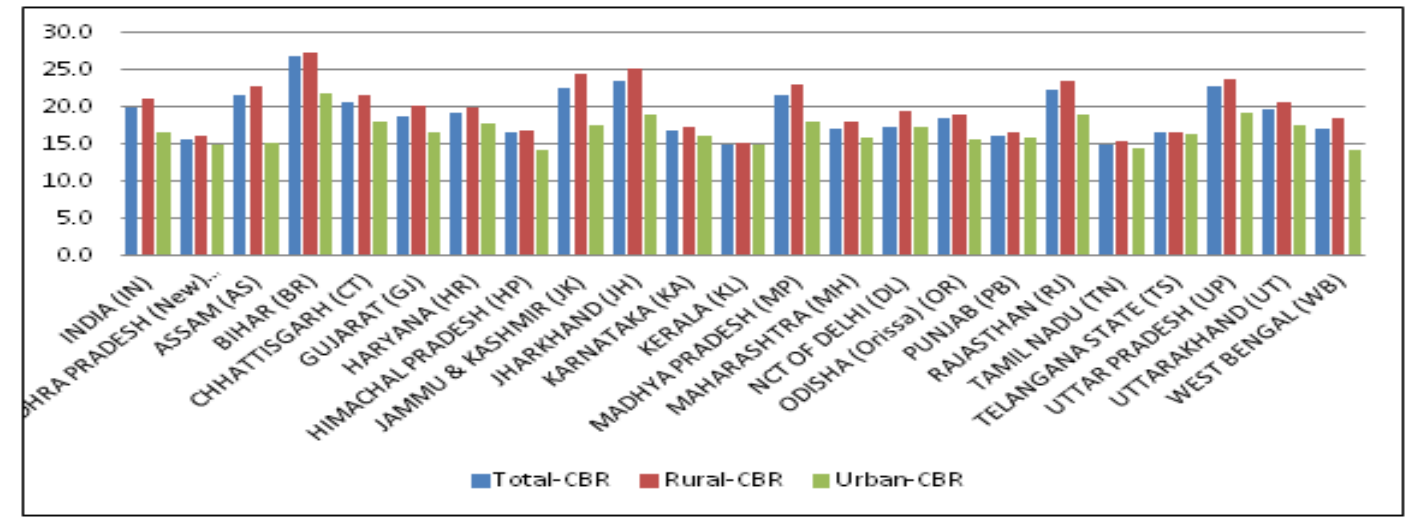

Figure 3. Indirect estimates of CBR - India and selected states-2007.

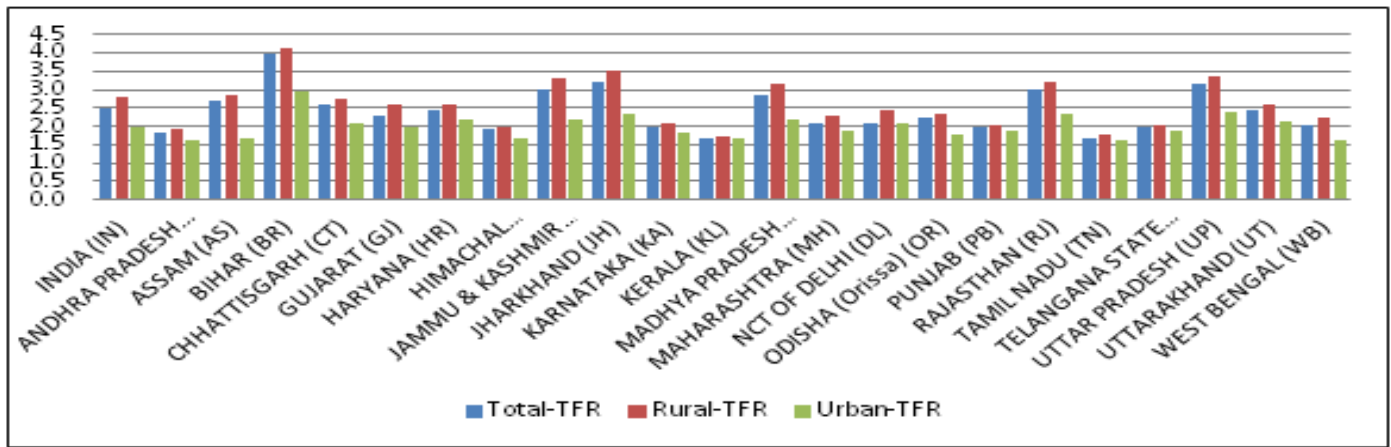

Figure 4. Indirect estimates of TFR -India and selected states-2007. 


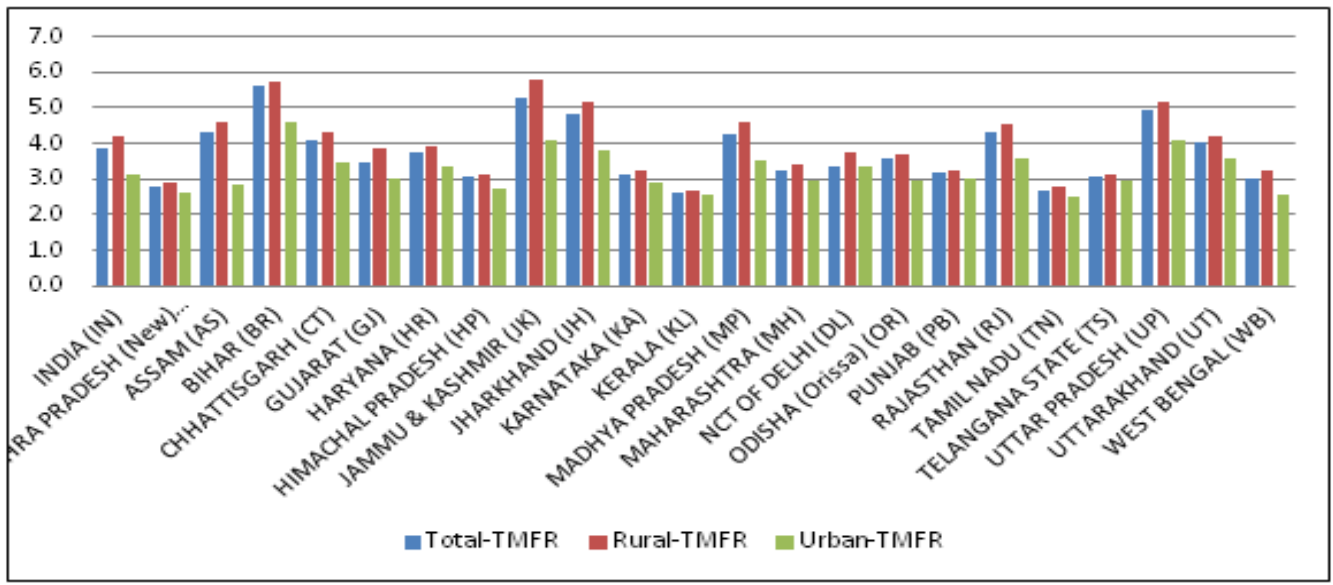

Figure 5. Indirect estimates of TMFR -India and selected states-2007.

Important conclusions that we may arrive at are: (1) whatever indicator you may consider, urban fertility is always observed to be less than the rural fertility. Interestingly, in case of Kerala for both rural and urban areas fertility remain to be the same due to obvious reasons. (2) States of north India especially Bihar, Jharkhand, Madhya Pradesh, Rajasthan and Uttar Pradesh always showed high fertility when compared to other states in India, irrespective of the fertility indicator you may consider. 3) A special mention has to be made here in case of the state of Jammu and Kashmir (JK). Guilmoto and Rajan (2013) also obtained almost similar results as observed here. While interpreting the result for JK the above authors pointed out that the reported age distribution of $\mathrm{JK}$ is not acceptable for fertility estimation due to obvious reason of low data quality. Thus to state, JK results at the state and district level provided here may also be accepted with a caution.

\section{CONCLUSION}

This study suggested a new and innovative methodology to estimate TFR and related measures using a simple ratio that is Child Women Ratio of ages 0-9. Using the age-sex marital status distribution of India data of 2011 census it provided the TFR, TMFR and related indirect estimates for India / States /UTs /Districts. Various estimates obviously refer to the year 2007, instead 2011 census year. A comparison of the estimates arrived at here, with that of Guilmoto and Rajan (2013) estimates of CBR and TFR derived using the 2011 age-sex distribution data indicates that they are quite comparable. As expected, urban fertility is observed to be lower than 
the rural fertility, among all districts in India. It is to conclude that the simple and innovative indirect method suggested here even it depends on few meaningful assumptions, may succeed in providing plausible estimates even for sub-state units, rural-urban regions, and religious groups, etc. if data permits. Thus the simple methodology presented here which use a simple ratio has wide implications and seems to be experimented further for its proper use and importance. The present methodology may be applicable to other developing countries in the world like that of Ethiopia, if (1) data permits and (2) the assumptions made also acceptable to some extent. The present method firstly allows estimation of fertility indicators even for the smallest units of analysis, secondly allows to test the accuracy of estimates arrived at by other methodologies, and thus seems to serve as a useful indirect technique for fertility analysis.

\section{ACKNOWLEDGEMENTS}

The authors are thankful to the anonymous reviewers for their constructive comments.

\section{REFERENCE}

Bhat, P. N. M. 1996. Contours of Fertility Decline in India: A District Level Study Based on the 1991 Census. In: K. Srinivasan (ed.) Population Policy and Reproductive Health, New Delhi: Hindustan Publishing Corporation, ISBN -10: 8170750423, pp.96-179.

Guilmoto, C. Z. \& Rajan, S. I. 2013. Fertility at the District Level in India: Lessons from the 2011 Census, Economic and Political weekly, EPW Vol. XLVIII No. 23(8):59-70 and Appendix tables on CBR and TFR.

Hauer, M., Baker, J \& Brown, W. 2013. Indirect Estimates of Total Fertility Rate Using Child Woman/Ratio: A Comparison with the Bogue-Palmore Method. PLOS ONE, 8(6):1-7, e67226 (From www.plosone.org).

Rele, J.R. 1976. Fertility Analysis through Extension of Stable Population Concepts. Westport, Connecticut: Greenwood Press. (Originally published in 1967 by International Population and Urban Research, University of California, Berkeley)

Rele, J.R. 1987. Fertility levels and trends in India, 1951-81. Population and Development Review, 13(3): 513-530.

Smith, D. P. 1992. Formal Demography. Plenum Press, ISBN: 978-0-306-43869-1, eBook ISBN: 978-1-4899-2465-0, pp.228-234. 


\begin{tabular}{|c|c|c|c|c|c|c|c|c|c|}
\hline \multirow[b]{2}{*}{ India/States/Uts/Districts } & \multicolumn{3}{|c|}{ TOTAL - 2007} & \multicolumn{3}{|c|}{ RURAL - 2007} & \multicolumn{3}{|c|}{ URBAN - 2007} \\
\hline & CBR & TFR & TMFR & CBR & TFR & TMFR & CBR & TFR & TMFR \\
\hline INDIA (IN) & 19.8 & 2.5 & 3.9 & 21.2 & 2.8 & 4.2 & 16.7 & 2.0 & 3.1 \\
\hline JAMMU \& KASHMIR (JK) & 22.5 & 3.0 & 5.3 & 24.4 & 3.3 & 5.8 & 17.5 & 2.2 & 4.1 \\
\hline Kupwara (JK) & 30.0 & 4.9 & 9.3 & 30.5 & 4.9 & 9.2 & 26.5 & 5.0 & 9.6 \\
\hline Badgam (JK) & 28.4 & 4.1 & 8.2 & 29.5 & 4.3 & 8.7 & 20.7 & 2.9 & 5.6 \\
\hline Leh(Ladakh)(JK) & 12.9 & 1.8 & 3.5 & 14.7 & 1.9 & 3.7 & 9.6 & 1.6 & 3.0 \\
\hline Kargil (JK) & 20.1 & 2.7 & 5.3 & 20.9 & 2.8 & 5.4 & 14.1 & 2.0 & 4.1 \\
\hline Punch (JK) & 24.8 & 3.5 & 5.6 & 25.5 & 3.6 & 5.7 & 16.8 & 2.3 & 4.0 \\
\hline Rajouri (JK) & 23.7 & 3.3 & 5.2 & 24.6 & 3.4 & 5.3 & 13.5 & 2.2 & 3.7 \\
\hline Kathua (JK) & 19.8 & 2.5 & 4.0 & 20.4 & 2.6 & 4.1 & 15.8 & 1.9 & 3.1 \\
\hline Baramula (JK) & 22.9 & 3.1 & 5.9 & 24.1 & 3.3 & 6.3 & 17.1 & 2.3 & 4.4 \\
\hline Bandipore (JK) & 22.7 & 3.1 & 6.0 & 23.7 & 3.2 & 6.3 & 17.7 & 2.2 & 4.5 \\
\hline Srinagar (JK) & 17.8 & 2.1 & 4.2 & 20.0 & 2.4 & 4.6 & 17.8 & 2.1 & 4.2 \\
\hline Ganderbal (JK) & 24.2 & 3.4 & 6.3 & 24.2 & 3.4 & 6.3 & 24.2 & 3.3 & 6.1 \\
\hline Pulwama (JK) & 22.8 & 2.9 & 5.6 & 22.9 & 2.9 & 5.6 & 21.9 & 3.0 & 6.0 \\
\hline Shupiyan (JK) & 22.1 & 2.8 & 5.2 & 22.4 & 2.8 & 5.2 & 16.7 & 2.2 & 4.3 \\
\hline Anantnag (JK) & 26.6 & 3.7 & 7.0 & 27.8 & 3.9 & 7.4 & 23.2 & 3.0 & 6.1 \\
\hline Kulgam (JK) & 23.1 & 2.9 & 5.5 & 23.8 & 3.1 & 5.7 & 19.9 & 2.5 & 4.6 \\
\hline Doda (JK) & 24.3 & 3.3 & 5.3 & 24.9 & 3.4 & 5.5 & 18.1 & 2.4 & 4.1 \\
\hline Ramban (JK) & 26.9 & 3.9 & 6.2 & 27.4 & 4.0 & 6.3 & 15.9 & 2.1 & 3.6 \\
\hline Kishtwar (JK) & 24.2 & 3.3 & 5.5 & 24.8 & 3.4 & 5.6 & 16.0 & 2.0 & 3.8 \\
\hline Udhampur (JK) & 21.7 & 2.9 & 4.4 & 23.3 & 3.1 & 4.7 & 14.9 & 2.0 & 3.2 \\
\hline Reasi (JK) & 24.9 & 3.5 & 5.6 & 25.6 & 3.7 & 5.7 & 17.6 & 2.2 & 3.7 \\
\hline Jammu (JK) & 15.9 & 1.9 & 3.1 & 17.8 & 2.1 & 3.5 & 14.0 & 1.7 & 2.7 \\
\hline Samba (JK) & 17.6 & 2.1 & 3.4 & 18.0 & 2.2 & 3.5 & 15.8 & 1.9 & 3.2 \\
\hline HIMACHAL PRADESH (HP) & 16.6 & 1.9 & 3.1 & 16.8 & 2.0 & 3.1 & 14.2 & 1.6 & 2.7 \\
\hline Chamba (HP) & 19.7 & 2.4 & 3.9 & 20.1 & 2.4 & 4.0 & 14.3 & 1.7 & 3.0 \\
\hline Kangra (HP) & 15.9 & 1.8 & 2.9 & 16.0 & 1.8 & 2.9 & 14.2 & 1.6 & 2.8 \\
\hline Lahul \& Spiti (HP) & 14.6 & 1.8 & 3.3 & 14.6 & 1.8 & 3.3 & NA & NA & NA \\
\hline Kullu (HP) & 16.8 & 2.0 & 3.2 & 17.0 & 2.0 & 3.2 & 14.5 & 1.7 & 2.7 \\
\hline Mandi (HP) & 16.5 & 1.9 & 2.9 & 16.7 & 1.9 & 2.9 & 13.3 & 1.5 & 2.4 \\
\hline Hamirpur (HP) & 15.7 & 1.8 & 2.7 & 15.8 & 1.8 & 2.7 & 13.8 & 1.5 & 2.5 \\
\hline Una (HP) & 16.6 & 2.0 & 3.2 & 16.7 & 2.0 & 3.2 & 15.8 & 1.9 & 3.1 \\
\hline Bilaspur (HP) & 15.8 & 1.9 & 2.9 & 15.9 & 1.9 & 2.9 & 14.7 & 1.7 & 2.7 \\
\hline Solan (HP) & 17.0 & 2.1 & 3.2 & 17.3 & 2.1 & 3.2 & 15.5 & 2.0 & 3.1 \\
\hline Sirmaur (HP) & 19.1 & 2.4 & 3.9 & 19.5 & 2.4 & 4.0 & 15.9 & 1.8 & 3.0 \\
\hline Shimla (HP) & 14.9 & 1.7 & 2.8 & 15.6 & 1.8 & 2.9 & 12.9 & 1.4 & 2.5 \\
\hline Kinnaur (HP) & 14.4 & 1.9 & 3.1 & 14.4 & 1.9 & 3.1 & NA & NA & NA \\
\hline PUNJAB (PB) & 16.2 & 2.0 & 3.2 & 16.5 & 2.0 & 3.3 & 15.8 & 1.9 & 3.0 \\
\hline Gurdaspur (PB) & 16.3 & 2.0 & 3.3 & 17.0 & 2.1 & 3.4 & 14.5 & 1.7 & 2.9 \\
\hline Kapurthala (PB) & 15.7 & 1.9 & 3.1 & 16.0 & 1.9 & 3.2 & 15.0 & 1.8 & 2.9 \\
\hline Jalandhar (PB) & 15.2 & 1.8 & 3.0 & 15.2 & 1.8 & 3.1 & 15.2 & 1.8 & 2.9 \\
\hline Hoshiarpur (PB) & 15.7 & 1.8 & 3.1 & 15.9 & 1.9 & 3.1 & 15.0 & 1.7 & 2.9 \\
\hline Shahid Bhagat Singh Nagar (PB) & 15.1 & 1.8 & 3.0 & 15.0 & 1.8 & 3.0 & 15.6 & 1.8 & 3.0 \\
\hline Fatehgarh Sahib (PB) & 15.4 & 1.9 & 3.0 & 15.1 & 1.8 & 2.9 & 16.0 & 1.9 & 3.1 \\
\hline Ludhiana (PB) & 16.1 & 1.9 & 3.1 & 15.4 & 1.9 & 3.1 & 16.5 & 2.0 & 3.1 \\
\hline Moga (PB) & 15.9 & 2.0 & 3.2 & 15.9 & 2.0 & 3.3 & 15.9 & 1.9 & 3.1 \\
\hline Firozpur (PB) & 17.8 & 2.2 & 3.5 & 18.3 & 2.3 & 3.6 & 16.5 & 2.0 & 3.2 \\
\hline Muktsar (PB) & 16.7 & 2.1 & 3.2 & 16.7 & 2.1 & 3.2 & 16.9 & 2.0 & 3.2 \\
\hline Faridkot (PB) & 16.4 & 2.0 & 3.2 & 16.4 & 2.0 & 3.2 & 16.3 & 2.0 & 3.1 \\
\hline Bathinda (PB) & 15.8 & 1.9 & 3.0 & 15.5 & 1.9 & 3.0 & 16.3 & 1.9 & 3.0 \\
\hline Mansa (PB) & 16.2 & 2.1 & 3.2 & 16.2 & 2.1 & 3.3 & 16.1 & 1.9 & 3.1 \\
\hline Patiala (PB) & 16.3 & 2.0 & 3.1 & 17.2 & 2.1 & 3.3 & 14.9 & 1.7 & 2.8 \\
\hline Amritsar (PB) & 16.5 & 2.0 & 3.2 & 18.0 & 2.2 & 3.6 & 15.2 & 1.8 & 2.9 \\
\hline Tarn Taran (PB) & 18.0 & 2.3 & 3.6 & 18.1 & 2.3 & 3.7 & 16.9 & 2.1 & 3.4 \\
\hline Rupnagar (PB) & 15.6 & 1.9 & 3.0 & 15.9 & 1.9 & 3.1 & 14.7 & 1.7 & 2.8 \\
\hline Sahibzada Ajit Singh Nagar (PB) & 16.8 & 2.0 & 3.1 & 17.1 & 2.1 & 3.2 & 16.5 & 1.9 & 3.0 \\
\hline Sangrur (PB) & 16.0 & 2.0 & 3.1 & 15.7 & 2.0 & 3.1 & 16.6 & 2.0 & 3.2 \\
\hline Barnala (PB) & 16.1 & 2.0 & 3.1 & 15.7 & 2.0 & 3.1 & 16.9 & 2.1 & 3.2 \\
\hline CHANDIGARH (CH) & 16.4 & 2.0 & 3.2 & 20.5 & 2.9 & 4.1 & 16.3 & 2.0 & 3.2 \\
\hline Chandigarh $(\mathrm{CH})$ & 16.4 & 2.0 & 3.2 & 20.5 & 2.9 & 4.1 & 16.3 & 2.0 & 3.2 \\
\hline UTTARAKHAND (UT) & 19.7 & 2.4 & 4.0 & 20.6 & 2.6 & 4.2 & 17.6 & 2.1 & 3.6 \\
\hline Uttarkashi (UT) & 20.7 & 2.7 & 4.2 & 21.1 & 2.7 & 4.3 & 16.7 & 2.0 & 3.3 \\
\hline Chamoli (UT) & 19.4 & 2.4 & 3.9 & 19.8 & 2.4 & 3.9 & 17.5 & 2.3 & 3.7 \\
\hline Rudraprayag (UT) & 19.6 & 2.3 & 3.7 & 19.8 & 2.3 & 3.7 & 16.1 & 2.2 & 3.5 \\
\hline Tehri Garhwal (UT) & 20.2 & 2.5 & 4.0 & 20.6 & 2.5 & 4.0 & 17.3 & 2.1 & 3.4 \\
\hline Dehradun (UT) & 17.4 & 2.1 & 3.5 & 19.3 & 2.4 & 4.0 & 15.8 & 1.9 & 3.1 \\
\hline Garhwal (UT) & 18.1 & 2.2 & 3.5 & 18.4 & 2.2 & 3.6 & 17.0 & 2.0 & 3.3 \\
\hline
\end{tabular}




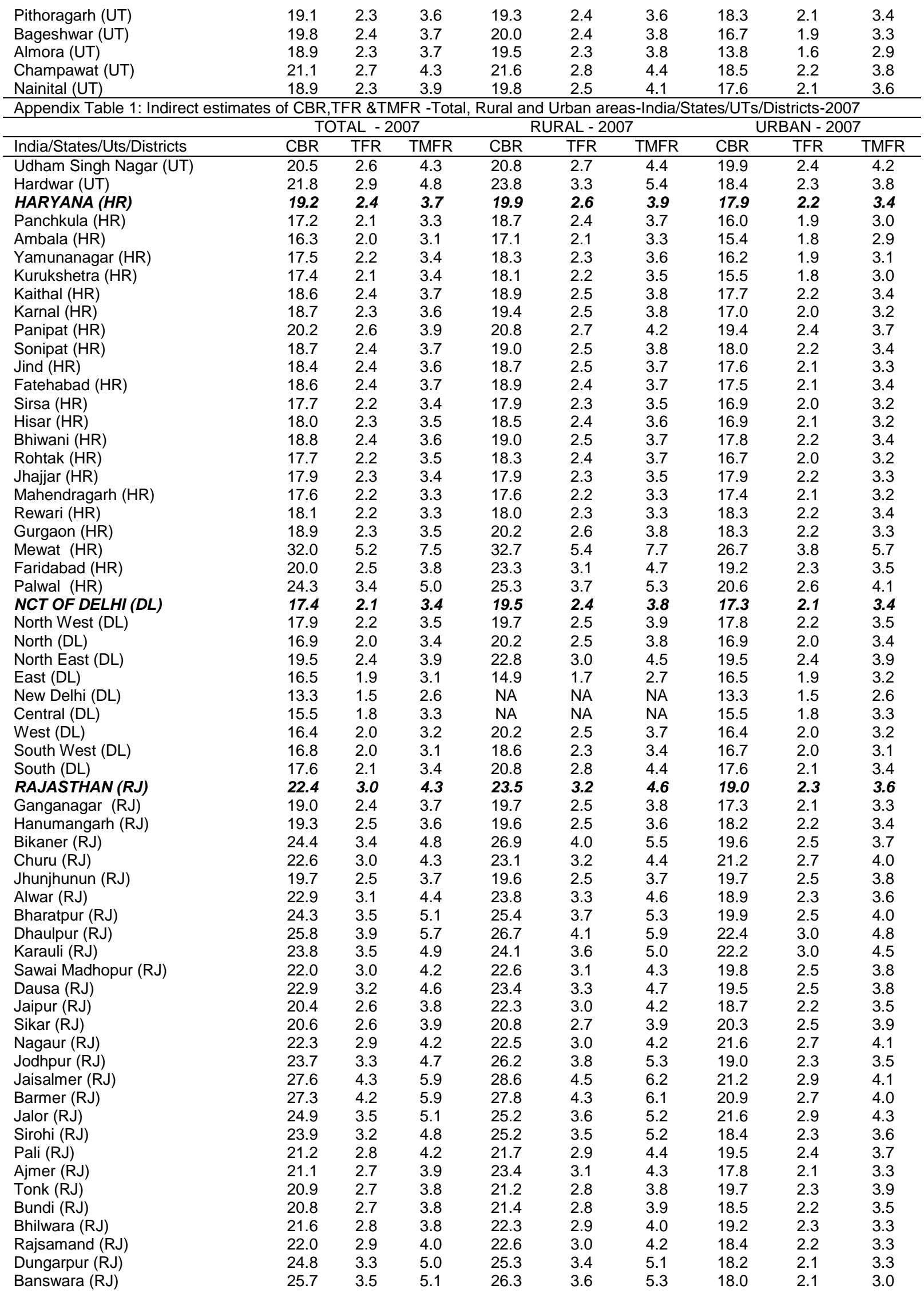




\begin{tabular}{|c|c|c|c|c|c|c|c|c|c|}
\hline Chittaurgarh (RJ) & 19.9 & 2.5 & 3.4 & 20.3 & 2.6 & 3.5 & 18.1 & 2.1 & 3.2 \\
\hline Kota (RJ) & 18.8 & 2.3 & 3.5 & 20.2 & 2.6 & 3.9 & 17.8 & 2.1 & 3.3 \\
\hline Baran (RJ) & 21.5 & 2.8 & 4.1 & 22.0 & 3.0 & 4.3 & 19.4 & 2.4 & 3.7 \\
\hline Jhalawar (RJ) & 21.4 & 2.8 & 3.9 & 21.7 & 2.9 & 3.9 & 19.4 & 2.4 & 3.6 \\
\hline Udaipur (RJ) & 23.6 & 3.1 & 4.5 & 25.4 & 3.5 & 4.9 & 16.1 & 1.9 & 2.9 \\
\hline Pratapgarh (RJ) & 24.7 & 3.3 & 4.7 & 25.3 & 3.4 & 4.9 & 17.7 & 2.1 & 3.2 \\
\hline UTTAR PRADESH (UP) & 22.7 & 3.1 & 4.9 & 23.8 & 3.4 & 5.2 & 19.1 & 2.4 & 4.1 \\
\hline Saharanpur (UP) & 21.8 & 2.9 & 4.9 & 22.7 & 3.1 & 5.2 & 19.8 & 2.5 & 4.4 \\
\hline Muzaffarnagar (ÚP) & 22.7 & 3.1 & 5.1 & 23.3 & 3.3 & 5.3 & 21.4 & 2.8 & 4.7 \\
\hline Bijnor (UP) & 22.5 & 3.0 & 5.2 & 22.8 & 3.1 & 5.3 & 21.5 & 2.8 & 5.2 \\
\hline \multicolumn{10}{|c|}{ Appendix Table 1: Indirect estimates of CBR,TFR \& TMFR -Total, Rural and Urban areas-India/States/UTs/Districts-2007 } \\
\hline & \multicolumn{3}{|c|}{ TOTAL - 2007} & \multicolumn{3}{|c|}{ RURAL - 2007} & \multicolumn{3}{|c|}{ URBAN - 2007} \\
\hline India/States/Uts/Districts & CBR & TFR & TMFR & CBR & TFR & TMFR & CBR & TFR & TMFR \\
\hline Moradabad (UP) & 24.1 & 3.4 & 5.8 & 25.7 & 3.8 & 6.2 & 20.8 & 2.7 & 4.8 \\
\hline Rampur (UP) & 24.0 & 3.4 & 5.8 & 25.2 & 3.7 & 6.1 & 20.4 & 2.6 & 4.9 \\
\hline Jyotiba Phule Nagar (UP) & 23.9 & 3.3 & 5.4 & 24.5 & 3.5 & 5.5 & 22.0 & 2.8 & 5.0 \\
\hline Meerut (UP) & 21.0 & 2.8 & 4.5 & 22.6 & 3.1 & 5.1 & 19.5 & 2.4 & 4.1 \\
\hline Baghpat (UP) & 21.7 & 3.0 & 4.8 & 21.7 & 3.1 & 4.9 & 21.5 & 2.8 & 4.6 \\
\hline Ghaziabad (UP) & 20.9 & 2.7 & 4.4 & 23.3 & 3.3 & 5.3 & 19.8 & 2.5 & 3.9 \\
\hline Gautam Buddha Nagar (UP) & 21.4 & 2.9 & 4.3 & 23.0 & 3.3 & 4.9 & 20.3 & 2.6 & 3.9 \\
\hline Bulandshahr (UP) & 22.6 & 3.1 & 5.0 & 23.0 & 3.2 & 5.1 & 21.2 & 2.7 & 4.6 \\
\hline Aligarh (UP) & 22.8 & 3.2 & 5.0 & 23.8 & 3.5 & 5.3 & 20.7 & 2.7 & 4.5 \\
\hline Mahamaya Nagar (UP) & 23.0 & 3.3 & 5.1 & 23.5 & 3.4 & 5.2 & 21.0 & 2.8 & 4.5 \\
\hline Mathura (UP) & 23.0 & 3.4 & 5.1 & 24.2 & 3.7 & 5.5 & 20.0 & 2.7 & 4.2 \\
\hline Agra (UP) & 22.3 & 3.0 & 4.8 & 24.8 & 3.6 & 5.4 & 19.3 & 2.4 & 4.0 \\
\hline Firozabad (UP) & 22.6 & 3.2 & 5.0 & 23.5 & 3.4 & 5.3 & 21.0 & 2.8 & 4.6 \\
\hline Mainpuri (UP) & 22.5 & 3.2 & 5.1 & 22.9 & 3.3 & 5.2 & 20.1 & 2.5 & 4.3 \\
\hline Budaun (UP) & 26.1 & 4.0 & 6.2 & 26.9 & 4.3 & 6.4 & 22.7 & 3.1 & 5.3 \\
\hline Bareilly (UP) & 23.2 & 3.3 & 5.4 & 25.2 & 3.8 & 6.0 & 19.6 & 2.6 & 4.5 \\
\hline Pilibhit (UP) & 22.4 & 3.1 & 5.1 & 23.2 & 3.3 & 5.2 & 19.0 & 2.4 & 4.3 \\
\hline Shahjahanpur (UP) & 24.6 & 3.6 & 5.6 & 25.8 & 4.0 & 5.9 & 19.5 & 2.5 & 4.4 \\
\hline Kheri (UP) & 24.3 & 3.5 & 5.4 & 24.9 & 3.7 & 5.5 & 19.7 & 2.5 & 4.2 \\
\hline Sitapur (UP) & 24.4 & 3.6 & 5.5 & 24.9 & 3.8 & 5.6 & 20.3 & 2.6 & 4.6 \\
\hline Hardoi (UP) & 23.5 & 3.5 & 5.4 & 24.1 & 3.7 & 5.5 & 20.0 & 2.6 & 4.4 \\
\hline Unnao (UP) & 20.9 & 2.9 & 4.7 & 21.4 & 3.1 & 4.9 & 18.3 & 2.3 & 3.9 \\
\hline Lucknow (ÚP) & 17.7 & 2.2 & 3.7 & 21.5 & 3.0 & 4.9 & 15.7 & 1.8 & 3.2 \\
\hline Rae Bareli (UP) & 21.2 & 2.8 & 4.6 & 21.7 & 2.9 & 4.7 & 17.0 & 2.0 & 3.5 \\
\hline Farrukhabad (UP) & 23.3 & 3.3 & 5.3 & 24.5 & 3.6 & 5.6 & 19.3 & 2.4 & 4.2 \\
\hline Kannauj (UP) & 22.9 & 3.3 & 5.4 & 23.2 & 3.4 & 5.5 & 21.3 & 2.8 & 4.9 \\
\hline Etawah (UP) & 21.0 & 2.9 & 4.5 & 21.9 & 3.1 & 4.8 & 18.0 & 2.2 & 3.7 \\
\hline Auraiya (UP) & 21.4 & 3.0 & 4.7 & 21.9 & 3.1 & 4.9 & 19.2 & 2.4 & 4.0 \\
\hline Kanpur Dehat (UP) & 20.5 & 2.9 & 4.6 & 20.7 & 2.9 & 4.7 & 19.2 & 2.5 & 4.1 \\
\hline Kanpur Nagar (UP) & 16.7 & 2.1 & 3.5 & 20.2 & 2.8 & 4.6 & 14.9 & 1.8 & 3.1 \\
\hline Jalaun (UP) & 20.0 & 2.7 & 4.1 & 20.6 & 2.9 & 4.2 & 18.2 & 2.3 & 3.7 \\
\hline Jhansi (UP) & 18.9 & 2.4 & 3.6 & 20.3 & 2.7 & 3.8 & 17.0 & 2.1 & 3.3 \\
\hline Lalitpur (UP) & 24.9 & 3.6 & 4.9 & 25.8 & 3.8 & 5.1 & 19.2 & 2.4 & 3.7 \\
\hline Hamirpur (UP) & 20.8 & 3.0 & 4.5 & 21.3 & 3.1 & 4.6 & 18.8 & 2.4 & 3.9 \\
\hline Mahoba (UP) & 21.8 & 3.2 & 4.7 & 22.3 & 3.3 & 4.8 & 19.8 & 2.7 & 4.2 \\
\hline Banda (UP) & 24.0 & 3.6 & 5.3 & 24.8 & 3.9 & 5.6 & 19.4 & 2.5 & 4.1 \\
\hline Chitrakoot (UP) & 25.7 & 3.9 & 5.7 & 26.2 & 4.1 & 5.9 & 20.5 & 2.7 & 4.4 \\
\hline Fatehpur (UP) & 22.1 & 3.1 & 4.9 & 22.5 & 3.2 & 5.1 & 18.8 & 2.3 & 4.0 \\
\hline Pratapgarh (UP) & 21.6 & 2.8 & 4.4 & 21.8 & 2.8 & 4.5 & 18.6 & 2.3 & 3.9 \\
\hline Kaushambi (UP) & 25.1 & 3.8 & 5.9 & 25.4 & 3.8 & 6.0 & 22.0 & 3.0 & 5.0 \\
\hline Allahabad (UP) & 22.1 & 3.0 & 4.8 & 24.0 & 3.4 & 5.2 & 16.4 & 2.0 & 3.5 \\
\hline Bara Banki (UP) & 23.5 & 3.4 & 5.4 & 23.8 & 3.5 & 5.5 & 20.3 & 2.7 & 4.7 \\
\hline Faizabad (UP) & 21.8 & 2.9 & 4.6 & 22.6 & 3.0 & 4.8 & 16.9 & 2.1 & 3.8 \\
\hline Ambedkar Nagar (UP) & 21.5 & 2.8 & 4.7 & 21.7 & 2.9 & 4.7 & 20.1 & 2.6 & 4.7 \\
\hline Sultanpur (UP) & 22.3 & 3.0 & 4.6 & 22.5 & 3.0 & 4.6 & 18.3 & 2.2 & 3.8 \\
\hline Bahraich (UP) & 27.1 & 4.1 & 5.9 & 27.7 & 4.2 & 6.0 & 21.0 & 2.7 & 4.7 \\
\hline Shrawasti (UP) & 27.3 & 4.1 & 5.5 & 27.4 & 4.1 & 5.6 & 22.5 & 3.0 & 4.9 \\
\hline Balrampur (UP) & 27.1 & 4.0 & 5.9 & 27.5 & 4.1 & 6.0 & 21.5 & 2.8 & 4.9 \\
\hline Gonda (UP) & 24.8 & 3.5 & 5.2 & 25.2 & 3.6 & 5.3 & 19.1 & 2.4 & 4.1 \\
\hline Siddharthnagar (UP) & 27.5 & 4.0 & 5.9 & 27.8 & 4.0 & 6.0 & 23.4 & 3.1 & 5.2 \\
\hline Basti (UP) & 23.7 & 3.2 & 5.1 & 24.1 & 3.3 & 5.1 & 17.8 & 2.2 & 3.7 \\
\hline Sant Kabir Nagar (UP) & 24.6 & 3.4 & 5.4 & 24.8 & 3.5 & 5.4 & 21.9 & 2.9 & 4.8 \\
\hline Mahrajganj (UP) & 23.8 & 3.3 & 4.8 & 24.0 & 3.4 & 4.9 & 19.7 & 2.5 & 4.1 \\
\hline Gorakhpur (UP) & 21.3 & 2.8 & 4.4 & 22.4 & 3.0 & 4.6 & 16.5 & 2.0 & 3.4 \\
\hline Kushinagar (UP) & 23.9 & 3.3 & 5.1 & 24.1 & 3.4 & 5.1 & 20.2 & 2.7 & 4.4 \\
\hline Deoria (UP) & 22.6 & 2.9 & 4.6 & 23.0 & 3.0 & 4.7 & 19.6 & 2.5 & 4.1 \\
\hline Azamgarh (UP) & 23.1 & 3.0 & 4.8 & 23.2 & 3.0 & 4.8 & 21.6 & 2.8 & 5.0 \\
\hline
\end{tabular}




\begin{tabular}{|c|c|c|c|c|c|c|c|c|c|}
\hline Mau (UP) & 23.4 & 3.2 & 5.2 & 23.4 & 3.2 & 5.0 & 23.3 & 3.1 & 5.7 \\
\hline Ballia (UP) & 22.1 & 3.0 & 4.7 & 22.3 & 3.1 & 4.7 & 19.8 & 2.6 & 4.2 \\
\hline Jaunpur (UP) & 22.7 & 3.0 & 4.6 & 22.9 & 3.0 & 4.6 & 20.3 & 2.5 & 4.4 \\
\hline Ghazipur (UP) & 23.1 & 3.2 & 4.9 & 23.3 & 3.3 & 5.0 & 20.7 & 2.7 & 4.6 \\
\hline Chandauli (UP) & 23.4 & 3.3 & 4.8 & 23.8 & 3.4 & 4.8 & 20.5 & 2.6 & 4.4 \\
\hline Varanasi (UP) & 20.3 & 2.7 & 4.2 & 22.1 & 3.0 & 4.5 & 17.9 & 2.2 & 3.8 \\
\hline \multicolumn{10}{|l|}{ Sant Rvidas Nagar (Bhadohi) } \\
\hline (UP) & 24.0 & 3.2 & 4.8 & 24.3 & 3.3 & 4.8 & 22.4 & 3.0 & 4.9 \\
\hline Mirzapur (UP) & 23.8 & 3.4 & 5.0 & 24.5 & 3.5 & 5.1 & 19.8 & 2.6 & 4.2 \\
\hline Sonbhadra (ÚP) & 25.2 & 3.6 & 5.1 & 26.6 & 3.9 & 5.5 & 18.3 & 2.2 & 3.5 \\
\hline Etah (UP) & 23.6 & 3.4 & 5.3 & 24.1 & 3.5 & 5.4 & 20.5 & 2.6 & 4.4 \\
\hline Kanshiram Nagar (UP) & 24.9 & 3.7 & 5.7 & 25.7 & 3.9 & 5.9 & 21.9 & 2.9 & 5.0 \\
\hline$B I H A R(B R)$ & 26.7 & 4.0 & 5.6 & 27.3 & 4.1 & 5.7 & 21.9 & 2.9 & 4.6 \\
\hline Pashchim Champaran (BR) & 28.6 & 4.4 & 6.1 & 29.1 & 4.5 & 6.2 & 23.9 & 3.3 & 5.1 \\
\hline Purba Champaran (BR) & 28.9 & 4.5 & 6.1 & 29.2 & 4.6 & 6.2 & 25.0 & 3.6 & 5.3 \\
\hline \multicolumn{10}{|c|}{ Appendix Table 1: Indirect estimates of CBR,TFR \&TMFR -Total, Rural and Urban areas-India/States/UTs/Districts-2007 } \\
\hline & \multicolumn{3}{|c|}{ TOTAL - 2007} & \multicolumn{3}{|c|}{ RURAL - 2007} & \multicolumn{3}{|c|}{ URBAN - 2007} \\
\hline India/States/Uts/Districts & CBR & TFR & TMFR & CBR & TFR & TMFR & CBR & TFR & TMFR \\
\hline Sheohar (BR) & 28.1 & 4.5 & 6.1 & 28.1 & 4.5 & 6.1 & 27.8 & 4.3 & 6.0 \\
\hline Sitamarhi (BR) & 27.9 & 4.3 & 6.0 & 28.2 & 4.4 & 6.0 & 23.4 & 3.3 & 4.9 \\
\hline Madhubani (BR) & 26.6 & 3.9 & 5.4 & 26.7 & 4.0 & 5.4 & 23.3 & 3.2 & 4.8 \\
\hline Supaul (BR) & 28.7 & 4.3 & 5.8 & 28.9 & 4.4 & 5.8 & 24.4 & 3.5 & 5.0 \\
\hline Araria (BR) & 29.6 & 4.5 & 6.3 & 30.0 & 4.6 & 6.3 & 24.3 & 3.4 & 5.1 \\
\hline Kishanganj (BR) & 29.3 & 4.4 & 6.8 & 29.9 & 4.5 & 7.0 & 24.1 & 3.3 & 5.2 \\
\hline Purnia (BR) & 28.8 & 4.4 & 6.3 & 29.6 & 4.6 & 6.5 & 22.6 & 3.1 & 4.8 \\
\hline Katihar (BR) & 28.9 & 4.4 & 6.3 & 29.6 & 4.5 & 6.5 & 21.5 & 2.8 & 4.5 \\
\hline Madhepura (BR) & 29.3 & 4.5 & 6.0 & 29.5 & 4.5 & 6.0 & 23.7 & 3.2 & 4.8 \\
\hline Saharsa (BR) & 29.3 & 4.5 & 6.0 & 29.7 & 4.6 & 6.1 & 23.8 & 3.3 & 4.8 \\
\hline Darbhanga (BR) & 26.7 & 4.0 & 5.6 & 27.2 & 4.1 & 5.7 & 21.8 & 2.9 & 4.5 \\
\hline Muzaffarpur (BR) & 25.5 & 3.8 & 5.4 & 26.1 & 3.9 & 5.5 & 19.7 & 2.5 & 4.0 \\
\hline Gopalganj (BR) & 25.9 & 3.6 & 5.3 & 26.1 & 3.6 & 5.4 & 23.7 & 3.2 & 5.0 \\
\hline Siwan (BR) & 24.6 & 3.4 & 5.3 & 24.8 & 3.5 & 5.3 & 20.8 & 2.7 & 4.6 \\
\hline Saran (BR) & 25.3 & 3.7 & 5.5 & 25.6 & 3.7 & 5.5 & 22.4 & 3.1 & 4.8 \\
\hline Vaishali (BR) & 24.8 & 3.8 & 5.2 & 25.0 & 3.8 & 5.3 & 22.2 & 3.1 & 4.7 \\
\hline Samastipur (BR) & 27.1 & 4.1 & 5.6 & 27.3 & 4.2 & 5.7 & 21.0 & 2.8 & 4.3 \\
\hline Begusarai (BR) & 26.8 & 4.1 & 5.6 & 27.4 & 4.2 & 5.7 & 24.6 & 3.5 & 5.1 \\
\hline Khagaria (BR) & 29.2 & 4.6 & 6.2 & 29.5 & 4.7 & 6.3 & 23.6 & 3.3 & 5.1 \\
\hline Bhagalpur (BR) & 26.1 & 3.9 & 5.7 & 27.2 & 4.2 & 5.9 & 21.6 & 2.9 & 4.7 \\
\hline Banka (BR) & 26.7 & 4.0 & 5.4 & 26.8 & 4.0 & 5.4 & 23.5 & 3.3 & 4.9 \\
\hline Munger (BR) & 24.4 & 3.5 & 5.1 & 25.9 & 3.9 & 5.5 & 20.4 & 2.7 & 4.3 \\
\hline Lakhisarai (BR) & 27.2 & 4.1 & 5.6 & 27.6 & 4.2 & 5.7 & 24.4 & 3.4 & 5.1 \\
\hline Sheikhpura (BR) & 27.6 & 4.1 & 5.8 & 28.2 & 4.3 & 5.8 & 25.2 & 3.6 & 5.3 \\
\hline Nalanda (BR) & 26.3 & 3.9 & 5.4 & 26.8 & 4.0 & 5.5 & 23.3 & 3.2 & 5.0 \\
\hline Patna (BR) & 23.5 & 3.3 & 4.9 & 26.7 & 4.0 & 5.6 & 19.4 & 2.5 & 4.0 \\
\hline Bhojpur (BR) & 24.7 & 3.6 & 5.2 & 25.3 & 3.7 & 5.2 & 21.6 & 2.9 & 4.7 \\
\hline Buxar (BR) & 25.2 & 3.7 & 5.3 & 25.6 & 3.8 & 5.4 & 21.5 & 2.9 & 4.6 \\
\hline Kaimur (Bhabua) (BR) & 26.8 & 4.0 & 5.6 & 27.0 & 4.1 & 5.7 & 22.2 & 3.1 & 4.7 \\
\hline Rohtas (BR) & 25.2 & 3.6 & 5.2 & 25.7 & 3.8 & 5.3 & 21.9 & 3.0 & 4.8 \\
\hline Aurangabad (BR) & 26.1 & 3.8 & 5.4 & 26.4 & 3.9 & 5.5 & 23.1 & 3.1 & 5.0 \\
\hline Gaya (BR) & 26.0 & 3.8 & 5.4 & 26.8 & 4.0 & 5.5 & 20.3 & 2.7 & 4.4 \\
\hline Nawada (BR) & 26.3 & 3.8 & 5.3 & 26.7 & 3.9 & 5.3 & 23.0 & 3.1 & 4.9 \\
\hline Jamui (BR) & 26.6 & 3.9 & 5.2 & 26.9 & 3.9 & 5.3 & 23.4 & 3.2 & 4.8 \\
\hline Jehanabad (BR) & 25.9 & 3.8 & 5.3 & 26.3 & 3.9 & 5.3 & 22.7 & 3.1 & 4.7 \\
\hline Arwal (BR) & 26.3 & 3.9 & 5.4 & 26.4 & 3.9 & 5.4 & 25.4 & 3.7 & 5.4 \\
\hline SIKKIM (SK) & 16.2 & 1.9 & 3.4 & 16.7 & 2.1 & 3.6 & 14.6 & 1.6 & 2.8 \\
\hline North District (SK) & 16.6 & 2.2 & 3.9 & 16.2 & 2.2 & 3.8 & 19.9 & 2.4 & 4.4 \\
\hline West District (SK) & 18.0 & 2.2 & 3.8 & 18.1 & 2.2 & 3.8 & 15.9 & 1.8 & 3.3 \\
\hline South District (SK) & 16.8 & 2.0 & 3.5 & 17.2 & 2.1 & 3.6 & 14.8 & 1.6 & 2.8 \\
\hline East District (SK) & 15.0 & 1.7 & 3.1 & 15.5 & 1.9 & 3.3 & 14.4 & 1.6 & 2.8 \\
\hline ARUNACHAL PRADESH (AR) & 22.7 & 2.9 & 5.0 & 23.8 & 3.1 & 5.3 & 18.9 & 2.2 & 3.9 \\
\hline Tawang (AR) & 17.3 & 2.5 & 4.9 & 19.7 & 2.7 & 5.3 & 9.0 & 1.9 & 3.3 \\
\hline West Kameng (AR) & 20.2 & 2.7 & 4.4 & 20.9 & 2.9 & 4.6 & 17.5 & 2.0 & 3.5 \\
\hline East Kameng (AR) & 27.9 & 3.7 & 6.0 & 28.1 & 3.7 & 6.0 & 27.1 & 3.6 & 6.1 \\
\hline Papum Pare (AR) & 21.1 & 2.4 & 4.3 & 23.4 & 2.9 & 5.0 & 19.3 & 2.1 & 3.8 \\
\hline Upper Subansiri (AR) & 22.8 & 2.7 & 4.9 & 23.2 & 2.8 & 5.0 & 20.6 & 2.5 & 4.5 \\
\hline West Siang (AR) & 20.1 & 2.5 & 4.4 & 20.9 & 2.7 & 4.7 & 17.5 & 2.1 & 3.6 \\
\hline East Siang (AR) & 19.4 & 2.3 & 4.2 & 20.0 & 2.4 & 4.5 & 17.9 & 2.0 & 3.5 \\
\hline Upper Siang (AR) & 20.9 & 2.9 & 4.9 & 20.9 & 3.0 & 5.1 & 20.9 & 2.5 & 4.3 \\
\hline Changlang (AR) & 24.9 & 3.3 & 5.4 & 26.0 & 3.5 & 5.7 & 17.4 & 2.1 & 3.5 \\
\hline Tirap (AR) & 26.2 & 3.6 & 6.1 & 27.7 & 4.0 & 6.6 & 19.5 & 2.4 & 4.2 \\
\hline
\end{tabular}




\begin{tabular}{|c|c|c|c|c|c|c|c|c|c|}
\hline Lower Subansiri (AR) & 19.2 & 2.2 & 4.4 & 19.5 & 2.2 & 4.5 & 17.2 & 1.8 & 3.7 \\
\hline Kurung Kumey (AR) & 27.7 & 3.5 & 6.0 & 27.7 & 3.5 & 6.0 & 28.3 & 3.9 & 6.7 \\
\hline Dibang Valley $(A R)$ & 20.0 & 2.6 & 4.9 & 20.6 & 2.8 & 5.1 & 18.8 & 2.4 & 4.6 \\
\hline Lower Dibang Valley (AR) & 22.0 & 2.7 & 4.7 & 23.5 & 3.0 & 5.0 & 16.3 & 1.9 & 3.5 \\
\hline Lohit (AR) & 23.8 & 3.1 & 5.0 & 25.1 & 3.4 & 5.4 & 19.5 & 2.3 & 4.0 \\
\hline Anjaw (AR) & 24.0 & 3.6 & 5.9 & 24.6 & 3.6 & 5.9 & 11.3 & 2.1 & 4.5 \\
\hline NAGALAND (NL) & 21.8 & 2.7 & 5.6 & 22.8 & 2.9 & 5.9 & 19.2 & 2.2 & 4.7 \\
\hline Mon (NL) & 23.8 & 3.2 & 6.4 & 24.0 & 3.2 & 6.5 & 22.4 & 2.8 & 5.7 \\
\hline Mokokchung (NL) & 16.3 & 1.9 & 4.2 & 16.5 & 2.0 & 4.3 & 15.5 & 1.9 & 3.9 \\
\hline Zunheboto (NL) & 21.5 & 2.7 & 5.7 & 21.9 & 2.7 & 5.7 & 20.1 & 2.5 & 5.8 \\
\hline Wokha (NL) & 18.3 & 2.1 & 4.7 & 18.9 & 2.2 & 4.8 & 16.1 & 1.8 & 4.2 \\
\hline Dimapur (NL) & 19.8 & 2.3 & 4.5 & 21.4 & 2.6 & 5.1 & 18.4 & 2.1 & 4.0 \\
\hline Phek (NL) & 24.8 & 3.2 & 6.5 & 25.5 & 3.3 & 6.6 & 20.9 & 2.7 & 5.5 \\
\hline Tuensang (NL) & 26.6 & 3.6 & 7.2 & 27.5 & 3.8 & 7.4 & 23.0 & 2.9 & 6.2 \\
\hline Longleng (NL) & 25.6 & 3.4 & 6.3 & 25.9 & 3.5 & 6.3 & 23.8 & 3.1 & 5.9 \\
\hline Kiphire (NL) & 28.6 & 3.9 & 7.0 & 29.1 & 4.0 & 6.9 & 26.5 & 3.5 & 7.1 \\
\hline Kohima (NL) & 20.1 & 2.4 & 5.5 & 21.4 & 2.7 & 6.1 & 18.5 & 2.1 & 4.8 \\
\hline Peren (NL) & 23.8 & 3.1 & 6.1 & 23.7 & 3.1 & 6.1 & 24.2 & 3.0 & 6.1 \\
\hline MANIPUR (MN) & 19.2 & 2.3 & 4.3 & 19.9 & 2.4 & 4.6 & 17.5 & 2.0 & 3.7 \\
\hline Senapati (MN) & 20.0 & 2.4 & 5.3 & 20.0 & 2.4 & 5.3 & 19.7 & 2.2 & 4.5 \\
\hline \multicolumn{10}{|c|}{ Appendix Table 1: Indirect estimates of CBR,TFR \&TMFR -Total, Rural and Urban areas-India/States/UTs/Districts-2007 } \\
\hline & \multicolumn{3}{|c|}{ TOTAL - 2007} & \multicolumn{3}{|c|}{ RURAL - 2007} & \multicolumn{3}{|c|}{ URBAN - 2007} \\
\hline India/States/Uts/Districts & CBR & TFR & TMFR & CBR & TFR & TMFR & CBR & TFR & TMFR \\
\hline Tamenglong (MN) & 20.2 & 2.4 & 5.1 & 20.1 & 2.4 & 5.1 & 20.8 & 2.5 & 5.0 \\
\hline Churachandpur (MN) & 19.7 & 2.3 & 4.6 & 19.9 & 2.4 & 4.7 & 16.8 & 1.9 & 3.8 \\
\hline Bishnupur (MN) & 19.1 & 2.3 & 4.0 & 19.5 & 2.3 & 4.1 & 18.5 & 2.2 & 3.9 \\
\hline Thoubal (MN) & 20.9 & 2.5 & 4.4 & 21.6 & 2.6 & 4.5 & 19.7 & 2.3 & 4.1 \\
\hline Imphal West (MN) & 17.1 & 1.9 & 3.5 & 18.7 & 2.2 & 3.8 & 16.1 & 1.8 & 3.4 \\
\hline Imphal East (MN) & 18.9 & 2.2 & 4.0 & 20.3 & 2.4 & 4.3 & 16.8 & 1.8 & 3.5 \\
\hline Ukhrul (MN) & 19.7 & 2.4 & 5.3 & 19.8 & 2.5 & 5.3 & 19.0 & 2.3 & 5.1 \\
\hline Chandel (ḾN) & 17.7 & 2.0 & 4.0 & 17.1 & 1.9 & 3.9 & 21.6 & 2.7 & 5.1 \\
\hline MIZORAM (MZ) & 21.8 & 2.6 & 5.1 & 25.1 & 3.3 & 5.8 & 18.7 & 2.1 & 4.5 \\
\hline Mamit (MZ) & 25.0 & 3.3 & 5.8 & 25.7 & 3.4 & 5.9 & 21.8 & 2.7 & 5.2 \\
\hline Kolasib (MZ) & 22.6 & 2.8 & 5.4 & 25.1 & 3.3 & 5.9 & 20.7 & 2.5 & 5.0 \\
\hline Aizawl (MZ) & 18.6 & 2.1 & 4.4 & 21.9 & 2.8 & 5.3 & 17.7 & 1.9 & 4.2 \\
\hline Champhai (MZ) & 23.3 & 2.9 & 5.6 & 24.4 & 3.2 & 5.8 & 21.6 & 2.6 & 5.2 \\
\hline Serchhip (MZ) & 20.6 & 2.5 & 5.2 & 22.4 & 2.8 & 5.7 & 18.8 & 2.2 & 4.7 \\
\hline Lunglei (MZ) & 22.3 & 2.8 & 5.2 & 25.7 & 3.4 & 5.9 & 17.8 & 2.1 & 4.3 \\
\hline Lawngtlai (MZ) & 26.8 & 3.5 & 5.9 & 28.0 & 3.8 & 6.0 & 20.9 & 2.5 & 5.1 \\
\hline Saiha (MZ) & 23.9 & 3.0 & 5.7 & 26.0 & 3.5 & 6.0 & 21.3 & 2.5 & 5.3 \\
\hline TRIPURA (TR) & 18.0 & 2.1 & 3.3 & 19.3 & 2.3 & 3.6 & 14.1 & 1.5 & 2.4 \\
\hline West Tripura (TR) & 16.0 & 1.8 & 2.8 & 17.4 & 2.0 & 3.2 & 13.8 & 1.5 & 2.4 \\
\hline South Tripura (TR) & 18.5 & 2.2 & 3.3 & 19.3 & 2.3 & 3.5 & 14.0 & 1.5 & 2.3 \\
\hline Dhalai (TR) & 21.4 & 2.7 & 4.2 & 22.0 & 2.8 & 4.3 & 16.2 & 1.8 & 2.9 \\
\hline North Tripura (TR) & 20.3 & 2.4 & 4.1 & 21.3 & 2.6 & 4.3 & 15.3 & 1.7 & 2.9 \\
\hline MEGHALAYA (ML) & 26.8 & 3.5 & 6.5 & 28.8 & 4.0 & 7.0 & 18.8 & 2.1 & 4.6 \\
\hline West Garo Hills (ML) & 24.9 & 3.2 & 5.5 & 25.9 & 3.4 & 5.7 & 17.3 & 1.9 & 3.9 \\
\hline East Garo Hills (ML) & 25.9 & 3.3 & 5.8 & 26.8 & 3.5 & 5.9 & 20.5 & 2.4 & 4.9 \\
\hline South Garo Hills (ML) & 27.1 & 3.7 & 6.4 & 27.4 & 3.8 & 6.4 & 23.6 & 3.0 & 5.7 \\
\hline West Khasi Hills (ML) & 31.3 & 4.6 & 8.3 & 31.6 & 4.7 & 8.3 & 29.1 & 3.9 & 8.1 \\
\hline Ribhoi (ML) & 28.6 & 4.0 & 7.1 & 29.2 & 4.0 & 7.2 & 23.3 & 3.2 & 6.3 \\
\hline East Khasi Hills (ML) & 23.6 & 2.9 & 6.0 & 28.8 & 3.9 & 7.7 & 17.2 & 1.9 & 4.1 \\
\hline Jaintia Hills (ML) & 31.6 & 4.5 & 8.3 & 32.5 & 4.7 & 8.5 & 19.7 & 2.2 & 5.0 \\
\hline ASSAM (AS) & 21.7 & 2.7 & 4.3 & 22.7 & 2.9 & 4.6 & 15.1 & 1.7 & 2.8 \\
\hline Kokrajhar (AS) & 22.4 & 2.8 & 4.4 & 22.9 & 2.9 & 4.5 & 14.7 & 1.6 & 2.7 \\
\hline Dhubri (AS) & 26.9 & 3.7 & 5.3 & 28.1 & 3.9 & 5.6 & 16.5 & 1.9 & 3.2 \\
\hline Goalpara (AS) & 24.4 & 3.1 & 4.8 & 25.0 & 3.3 & 5.0 & 20.4 & 2.4 & 3.9 \\
\hline Barpeta (AS) & 24.7 & 3.3 & 4.8 & 25.6 & 3.5 & 5.0 & 14.6 & 1.6 & 2.8 \\
\hline Morigaon (AS) & 24.9 & 3.3 & 5.0 & 25.5 & 3.4 & 5.2 & 17.4 & 2.0 & 3.2 \\
\hline Nagaon (AS) & 23.7 & 3.1 & 4.8 & 24.9 & 3.3 & 5.1 & 15.7 & 1.8 & 3.0 \\
\hline Sonitpur (AS) & 21.2 & 2.6 & 4.2 & 21.8 & 2.7 & 4.4 & 14.4 & 1.6 & 2.7 \\
\hline Lakhimpur (AS) & 21.9 & 2.7 & 4.3 & 22.4 & 2.8 & 4.5 & 17.0 & 1.9 & 3.1 \\
\hline Dhemaji (AS) & 22.3 & 2.8 & 4.4 & 22.6 & 2.9 & 4.5 & 18.0 & 2.1 & 3.4 \\
\hline Tinsukia (AS) & 20.2 & 2.4 & 4.0 & 21.5 & 2.6 & 4.4 & 14.9 & 1.7 & 2.8 \\
\hline Dibrugarh (AS) & 18.2 & 2.1 & 3.5 & 19.1 & 2.2 & 3.8 & 13.9 & 1.5 & 2.6 \\
\hline Sivasagar (AS) & 18.0 & 2.1 & 3.4 & 18.3 & 2.1 & 3.5 & 14.9 & 1.7 & 2.7 \\
\hline Jorhat (AS) & 16.9 & 1.9 & 3.2 & 17.5 & 2.0 & 3.4 & 14.2 & 1.6 & 2.6 \\
\hline Golaghat (AS) & 18.7 & 2.2 & 3.6 & 19.1 & 2.3 & 3.7 & 14.6 & 1.6 & 2.7 \\
\hline Karbi Anglong (AS) & 23.1 & 2.9 & 4.9 & 23.8 & 3.0 & 5.1 & 17.4 & 1.9 & 3.5 \\
\hline Dima Hasao (AS) & 21.8 & 2.7 & 4.9 & 23.7 & 3.0 & 5.5 & 17.3 & 2.0 & 3.6 \\
\hline
\end{tabular}




\begin{tabular}{|c|c|c|c|c|c|c|c|c|c|}
\hline Cachar (AS) & 21.3 & 2.6 & 4.5 & 22.5 & 2.8 & 4.9 & 15.9 & 1.8 & 3.1 \\
\hline Karimganj (AS) & 24.6 & 3.2 & 5.5 & 25.5 & 3.4 & 5.8 & 15.6 & 1.7 & 3.2 \\
\hline Hailakandi (AS) & 24.4 & 3.2 & 5.5 & 25.1 & 3.3 & 5.7 & 14.5 & 1.6 & 2.9 \\
\hline Bongaigaon (AS) & 22.8 & 2.9 & 4.4 & 24.2 & 3.1 & 4.7 & 14.9 & 1.6 & 2.7 \\
\hline Chirang (AS) & 22.4 & 2.8 & 4.4 & 22.8 & 2.8 & 4.5 & 17.1 & 2.0 & 3.2 \\
\hline Kamrup (AS) & 19.5 & 2.4 & 3.8 & 20.0 & 2.5 & 3.9 & 14.0 & 1.5 & 2.7 \\
\hline Kamrup Metropolitan (AS) & 14.7 & 1.6 & 2.7 & 18.6 & 2.2 & 3.6 & 13.9 & 1.5 & 2.5 \\
\hline Nalbari (AS) & 18.2 & 2.2 & 3.6 & 18.7 & 2.3 & 3.7 & 13.8 & 1.5 & 2.6 \\
\hline Baksa (AS) & 19.3 & 2.3 & 3.7 & 19.3 & 2.3 & 3.7 & 18.2 & 2.2 & 3.4 \\
\hline Darrang (AS) & 24.6 & 3.2 & 4.9 & 25.2 & 3.4 & 5.0 & 15.1 & 1.7 & 2.8 \\
\hline Udalguri (AS) & 20.2 & 2.4 & 4.0 & 20.5 & 2.5 & 4.1 & 14.6 & 1.6 & 2.7 \\
\hline WEST BENGAL (WB) & 17.1 & 2.0 & 3.0 & 18.5 & 2.2 & 3.3 & 14.1 & 1.6 & 2.5 \\
\hline Darjiling (WB) & 15.9 & 1.8 & 3.0 & 17.0 & 1.9 & 3.3 & 14.1 & 1.5 & 2.6 \\
\hline Jalpaiguri (WB) & 17.6 & 2.0 & 3.3 & 18.3 & 2.1 & 3.5 & 15.5 & 1.8 & 2.8 \\
\hline Koch Bihar (WB) & 17.9 & 2.2 & 3.2 & 18.5 & 2.2 & 3.3 & 13.0 & 1.4 & 2.3 \\
\hline Uttar Dinajpur (WB) & 23.9 & 3.2 & 4.9 & 24.7 & 3.4 & 5.2 & 18.1 & 2.2 & 3.5 \\
\hline Dakshin Dinajpur (WB) & 17.0 & 2.0 & 3.0 & 17.8 & 2.1 & 3.1 & 12.4 & 1.4 & 2.1 \\
\hline Maldah (WB) & 22.3 & 2.9 & 4.3 & 22.7 & 3.0 & 4.4 & 20.0 & 2.5 & 3.8 \\
\hline Murshidabad (WB) & 20.9 & 2.6 & 3.8 & 20.9 & 2.6 & 3.8 & 20.9 & 2.6 & 4.1 \\
\hline Birbhum (WB) & 18.6 & 2.2 & 3.2 & 19.1 & 2.3 & 3.3 & 15.5 & 1.7 & 2.7 \\
\hline Barddhaman (WB) & 15.8 & 1.8 & 2.7 & 16.2 & 1.9 & 2.7 & 15.3 & 1.7 & 2.7 \\
\hline Nadia (WB) & 15.2 & 1.8 & 2.6 & 16.2 & 1.9 & 2.8 & 12.4 & 1.4 & 2.1 \\
\hline North 24 Parganas (WB) & 14.3 & 1.6 & 2.5 & 17.0 & 2.0 & 2.9 & 12.3 & 1.4 & 2.1 \\
\hline Hugli (WB) & 14.2 & 1.6 & 2.4 & 15.1 & 1.7 & 2.5 & 12.9 & 1.5 & 2.3 \\
\hline Bankura (WB) & 17.0 & 2.1 & 3.0 & 17.3 & 2.1 & 3.0 & 13.3 & 1.5 & 2.3 \\
\hline \multirow{2}{*}{\multicolumn{10}{|c|}{$\begin{array}{r}\text { Appendix Table 1: Indirect estimates of CBR,TFR \&TMFR -Tota } \\
\text { TOTAL }-2007\end{array}$}} \\
\hline & & & & & & & & & \\
\hline India/States/Uts/Districts & CBR & TFR & TMFR & CBR & TFR & TMFR & CBR & TFR & TMFR \\
\hline Puruliya (WB) & 20.2 & 2.6 & 3.8 & 20.6 & 2.7 & 3.9 & 17.6 & 2.1 & 3.3 \\
\hline Haora (WB) & 15.7 & 1.8 & 2.8 & 16.8 & 2.0 & 2.9 & 15.1 & 1.7 & 2.7 \\
\hline Kolkata (WB) & 11.4 & 1.3 & 2.2 & NA & NA & NA & 11.4 & 1.3 & 2.2 \\
\hline South 24 Parganas (WB) & 18.5 & 2.2 & 3.2 & 19.5 & 2.4 & 3.4 & 15.4 & 1.7 & 2.6 \\
\hline Paschim Medinipur (WB) & 17.0 & 2.0 & 2.9 & 17.4 & 2.1 & 3.0 & 13.9 & 1.5 & 2.5 \\
\hline Purba Medinipur (WB) & 17.0 & 2.0 & 2.9 & 17.1 & 2.0 & 2.9 & 15.8 & 1.8 & 2.8 \\
\hline JHARKHAND (JH) & 23.6 & 3.2 & 4.8 & 25.1 & 3.5 & 5.1 & 18.9 & 2.3 & 3.8 \\
\hline Garhwa (JH) & 26.3 & 4.0 & 5.5 & 26.6 & 4.0 & 5.6 & 21.8 & 3.0 & 4.5 \\
\hline Chatra $(\mathrm{JH})$ & 27.2 & 3.9 & 5.5 & 27.5 & 4.0 & 5.6 & 21.9 & 3.0 & 4.8 \\
\hline Kodarma (JH) & 26.3 & 3.7 & 5.2 & 27.2 & 3.9 & 5.3 & 22.8 & 3.1 & 4.5 \\
\hline Giridih (JH) & 26.9 & 3.9 & 5.2 & 27.3 & 4.0 & 5.3 & 22.0 & 2.8 & 4.5 \\
\hline Deoghar (JH) & 25.5 & 3.6 & 4.9 & 26.7 & 3.9 & 5.2 & 19.7 & 2.5 & 3.9 \\
\hline Godda (JH) & 26.2 & 3.8 & 5.3 & 26.5 & 3.8 & 5.3 & 21.3 & 2.8 & 4.2 \\
\hline Sahibganj (JH) & 27.5 & 3.9 & 5.7 & 28.3 & 4.1 & 5.8 & 22.6 & 3.1 & 4.8 \\
\hline Pakur (JH) & 27.9 & 3.8 & 5.6 & 28.2 & 3.9 & 5.7 & 24.0 & 3.2 & 5.1 \\
\hline Dhanbad (JH) & 20.4 & 2.7 & 4.2 & 22.3 & 3.0 & 4.5 & 19.1 & 2.4 & 4.0 \\
\hline Bokaro (JH) & 20.9 & 2.7 & 4.1 & 22.9 & 3.1 & 4.5 & 18.6 & 2.3 & 3.7 \\
\hline Lohardaga (JH) & 24.8 & 3.4 & 5.5 & 25.5 & 3.6 & 5.7 & 20.1 & 2.5 & 4.4 \\
\hline Purbi Singhbhum (JH) & 18.7 & 2.2 & 3.6 & 20.7 & 2.6 & 4.1 & 17.0 & 2.0 & 3.3 \\
\hline Palamu (JH) & 24.9 & 3.6 & 5.2 & 25.5 & 3.8 & 5.3 & 20.5 & 2.7 & 4.3 \\
\hline Latehar (JH) & 27.2 & 3.9 & 6.0 & 27.6 & 4.0 & 6.1 & 21.8 & 2.9 & 4.7 \\
\hline Hazaribagh (JH) & 23.7 & 3.2 & 4.7 & 24.6 & 3.4 & 4.9 & 18.9 & 2.3 & 3.9 \\
\hline Ramgarh (JH) & 20.8 & 2.7 & 4.2 & 22.0 & 2.9 & 4.4 & 19.2 & 2.4 & 3.9 \\
\hline Dumka (JH) & 23.7 & 3.2 & 4.6 & 24.1 & 3.2 & 4.6 & 18.8 & 2.4 & 3.7 \\
\hline Jamtara (JH) & 23.7 & 3.2 & 4.6 & 24.2 & 3.3 & 4.7 & 18.8 & 2.4 & 3.7 \\
\hline Ranchi (JH) & 20.2 & 2.6 & 4.2 & 22.3 & 3.0 & 4.8 & 17.5 & 2.1 & 3.6 \\
\hline Khunti (JH) & 23.3 & 3.1 & 5.1 & 23.8 & 3.2 & 5.2 & 19.0 & 2.3 & 4.0 \\
\hline Gumla (JH) & 24.7 & 3.4 & 5.7 & 25.0 & 3.5 & 5.7 & 19.6 & 2.4 & 4.4 \\
\hline Simdega $(\mathrm{JH})$ & 22.6 & 3.0 & 5.2 & 22.8 & 3.1 & 5.3 & 19.0 & 2.3 & 4.4 \\
\hline Pashchimi Singhbhum (JH) & 24.9 & 3.2 & 5.6 & 26.1 & 3.5 & 5.9 & 18.4 & 2.2 & 3.9 \\
\hline Saraikela-Kharsawan (JH) & 21.6 & 2.8 & 4.5 & 21.9 & 2.9 & 4.7 & 20.7 & 2.5 & 4.0 \\
\hline ODISHA (Orissa) (OR) & 18.4 & 2.2 & 3.6 & 19.0 & 2.3 & 3.7 & 15.7 & 1.8 & 2.9 \\
\hline Bargarh (OR) & 15.6 & 1.8 & 3.0 & 15.6 & 1.8 & 3.0 & 16.0 & 1.8 & 3.0 \\
\hline Jharsuguda (OR) & 16.1 & 1.9 & 3.2 & 15.5 & 1.8 & 3.2 & 17.1 & 2.0 & 3.3 \\
\hline Sambalpur (OR) & 16.4 & 1.9 & 3.3 & 16.7 & 2.0 & 3.4 & 15.7 & 1.7 & 2.9 \\
\hline Debagarh (OR) & 18.4 & 2.2 & 3.6 & 18.5 & 2.3 & 3.7 & 17.0 & 2.0 & 3.4 \\
\hline Sundargarh (OR) & 18.5 & 2.2 & 3.7 & 19.8 & 2.4 & 4.1 & 16.2 & 1.8 & 3.1 \\
\hline Kendujhar (OR) & 20.8 & 2.6 & 4.1 & 21.1 & 2.6 & 4.2 & 19.2 & 2.3 & 3.8 \\
\hline Mayurbhanj (OR) & 20.7 & 2.6 & 4.1 & 21.1 & 2.7 & 4.2 & 15.0 & 1.7 & 2.8 \\
\hline Baleshwar (OR) & 18.3 & 2.2 & 3.4 & 18.6 & 2.3 & 3.5 & 16.2 & 1.8 & 2.9 \\
\hline Bhadrak (OR) & 17.8 & 2.1 & 3.5 & 17.7 & 2.1 & 3.5 & 18.0 & 2.1 & 3.6 \\
\hline Kendrapara (OR) & 16.4 & 1.9 & 3.2 & 16.4 & 2.0 & 3.2 & 16.0 & 1.9 & 3.2 \\
\hline
\end{tabular}




\begin{tabular}{|c|c|c|c|c|c|c|c|c|c|}
\hline Jagatsinghapur (OR) & 14.3 & 1.7 & 2.7 & 14.2 & 1.7 & 2.7 & 15.3 & 1.7 & 2.8 \\
\hline Cuttack (OR) & 15.1 & 1.8 & 2.9 & 15.6 & 1.9 & 3.0 & 13.8 & 1.5 & 2.6 \\
\hline Jajapur (OR) & 17.1 & 2.0 & 3.4 & 17.1 & 2.0 & 3.4 & 17.5 & 2.0 & 3.3 \\
\hline Dhenkanal (OR) & 17.0 & 2.0 & 3.2 & 17.2 & 2.1 & 3.3 & 14.8 & 1.7 & 2.8 \\
\hline Anugul (OR) & 17.5 & 2.1 & 3.3 & 17.8 & 2.2 & 3.4 & 15.5 & 1.7 & 2.8 \\
\hline Nayagarh (OR) & 16.4 & 2.1 & 3.0 & 16.5 & 2.1 & 3.0 & 14.9 & 1.8 & 2.7 \\
\hline Khordha (OR) & 15.6 & 1.8 & 2.9 & 16.5 & 2.0 & 3.1 & 14.6 & 1.6 & 2.6 \\
\hline Puri (OR) & 15.2 & 1.8 & 2.9 & 15.3 & 1.8 & 3.0 & 14.3 & 1.6 & 2.7 \\
\hline Ganjam (OR) & 17.8 & 2.2 & 3.4 & 18.6 & 2.3 & 3.6 & 14.9 & 1.7 & 2.9 \\
\hline Gajapati (OR) & 22.6 & 2.8 & 4.6 & 23.5 & 3.0 & 4.8 & 15.6 & 1.8 & 3.0 \\
\hline Kandhamal (OR) & 22.6 & 2.9 & 4.8 & 23.2 & 3.0 & 4.9 & 16.8 & 1.9 & 3.3 \\
\hline Baudh (OR) & 20.3 & 2.6 & 4.1 & 20.5 & 2.6 & 4.1 & 15.5 & 1.8 & 3.1 \\
\hline Subarnapur (OR) & 17.5 & 2.2 & 3.6 & 17.6 & 2.2 & 3.7 & 15.9 & 1.9 & 3.1 \\
\hline Balangir (OR) & 18.7 & 2.4 & 3.8 & 19.1 & 2.4 & 3.9 & 15.9 & 1.8 & 3.0 \\
\hline Nuapada (OR) & 20.9 & 2.7 & 4.1 & 21.0 & 2.7 & 4.2 & 18.2 & 2.1 & 3.5 \\
\hline Kalahandi (OR) & 20.5 & 2.6 & 4.0 & 20.9 & 2.7 & 4.0 & 16.8 & 1.9 & 3.2 \\
\hline Rayagada (OR) & 23.3 & 2.9 & 4.7 & 24.5 & 3.1 & 5.1 & 16.3 & 1.8 & 3.0 \\
\hline Nabarangapur (OR) & 24.8 & 3.3 & 4.8 & 25.3 & 3.4 & 4.9 & 18.4 & 2.2 & 3.6 \\
\hline Koraput (OR) & 23.9 & 3.1 & 4.7 & 25.3 & 3.3 & 5.0 & 17.1 & 1.9 & 3.2 \\
\hline Malkangiri (OR) & 26.0 & 3.5 & 5.3 & 26.5 & 3.6 & 5.4 & 20.1 & 2.5 & 4.1 \\
\hline CHHATTISGARH (CT) & 20.7 & 2.6 & 4.1 & 21.6 & 2.7 & 4.3 & 17.9 & 2.1 & 3.5 \\
\hline Koriya $(\mathrm{CT})$ & 20.8 & 2.6 & 4.0 & 22.8 & 2.9 & 4.4 & 16.6 & 1.9 & 3.2 \\
\hline Surguja (CT) & 23.2 & 3.1 & 4.6 & 23.8 & 3.2 & 4.7 & 18.5 & 2.2 & 3.6 \\
\hline Jashpur (CT) & 20.5 & 2.6 & 4.1 & 20.6 & 2.6 & 4.2 & 18.7 & 2.2 & 3.9 \\
\hline Raigarh (CT) & 18.9 & 2.3 & 3.6 & 19.1 & 2.4 & 3.7 & 18.0 & 2.1 & 3.5 \\
\hline Korba (CT) & 20.4 & 2.5 & 4.0 & 21.7 & 2.8 & 4.3 & 18.1 & 2.1 & 3.4 \\
\hline Janjgir - Champa (CT) & 20.2 & 2.6 & 4.3 & 20.4 & 2.7 & 4.3 & 18.7 & 2.2 & 3.8 \\
\hline Bilaspur (CT) & 22.1 & 2.9 & 4.5 & 23.5 & 3.1 & 4.8 & 18.2 & 2.1 & 3.6 \\
\hline Kabeerdham (CT) & 24.7 & 3.3 & 4.8 & 25.1 & 3.3 & 4.8 & 21.1 & 2.6 & 4.1 \\
\hline Rajnandgaon (CT) & 19.9 & 2.4 & 3.9 & 20.5 & 2.5 & 4.0 & 17.0 & 2.0 & 3.4 \\
\hline \multicolumn{10}{|c|}{ Appendix Table 1: Indirect estimates of CBR,TFR \&TMFR -Total, Rural and Urban areas-India/States/UTs/Districts-2007 } \\
\hline & \multicolumn{3}{|c|}{ TOTAL - 2007} & \multicolumn{3}{|c|}{ RURAL - 2007} & \multicolumn{3}{|c|}{ URBAN - 2007} \\
\hline India/States/Uts/Districts & CBR & TFR & TMFR & CBR & TFR & TMFR & CBR & TFR & TMFR \\
\hline Durg (CT) & 18.7 & 2.2 & 3.6 & 19.9 & 2.5 & 3.8 & 16.7 & 1.9 & 3.1 \\
\hline Raipur (CT) & 20.5 & 2.5 & 4.0 & 21.4 & 2.7 & 4.3 & 18.9 & 2.2 & 3.6 \\
\hline Mahasamund (CT) & 18.7 & 2.3 & 3.6 & 18.7 & 2.3 & 3.6 & 18.1 & 2.1 & 3.6 \\
\hline Dhamtari (CT) & 18.5 & 2.2 & 3.6 & 18.8 & 2.3 & 3.6 & 17.0 & 1.9 & 3.3 \\
\hline Uttar Bastar Kanker (CT) & 19.6 & 2.4 & 4.0 & 20.0 & 2.4 & 4.0 & 16.6 & 1.8 & 3.3 \\
\hline Bastar (CT) & 22.3 & 2.7 & 4.5 & 23.2 & 2.9 & 4.7 & 16.8 & 1.9 & 3.4 \\
\hline Narayanpur (CT) & 25.6 & 3.3 & 5.8 & 27.0 & 3.6 & 6.1 & 17.9 & 2.1 & 4.0 \\
\hline Dakshin Bastar Dantewada (CT) & 23.1 & 2.8 & 4.8 & 23.9 & 2.9 & 5.0 & 19.8 & 2.4 & 4.0 \\
\hline Bijapur (CT) & 26.3 & 3.3 & 5.6 & 26.8 & 3.4 & 5.7 & 21.9 & 2.7 & 4.8 \\
\hline MADHYA PRADESH (MP) & 21.7 & 2.9 & 4.3 & 23.0 & 3.1 & 4.6 & 18.1 & 2.2 & 3.5 \\
\hline Sheopur (MP) & 24.3 & 3.4 & 4.8 & 25.0 & 3.5 & 4.9 & 21.0 & 2.7 & 4.3 \\
\hline Morena (MP) & 22.3 & 3.1 & 4.5 & 22.9 & 3.3 & 4.7 & 20.3 & 2.6 & 4.0 \\
\hline Bhind (MP) & 21.0 & 2.9 & 4.3 & 21.4 & 3.1 & 4.4 & 19.8 & 2.6 & 3.9 \\
\hline Gwalior (MP) & 18.9 & 2.4 & 3.7 & 22.3 & 3.1 & 4.6 & 16.8 & 2.0 & 3.3 \\
\hline Datia (MP) & 20.5 & 2.8 & 4.0 & 21.0 & 2.9 & 4.1 & 18.9 & 2.4 & 3.8 \\
\hline Shivpuri (MP) & 24.1 & 3.5 & 4.9 & 25.0 & 3.7 & 5.1 & 19.9 & 2.5 & 4.0 \\
\hline Tikamgarh (MP) & 23.0 & 3.2 & 4.4 & 23.6 & 3.3 & 4.5 & 20.2 & 2.6 & 4.0 \\
\hline Chhatarpur (MP) & 23.4 & 3.4 & 4.9 & 24.5 & 3.7 & 5.2 & 19.8 & 2.6 & 4.1 \\
\hline Panna (MP) & 23.5 & 3.4 & 5.0 & 24.2 & 3.5 & 5.1 & 19.0 & 2.4 & 4.0 \\
\hline Sagar (MP) & 21.7 & 3.0 & 4.5 & 23.1 & 3.3 & 4.8 & 18.6 & 2.3 & 3.9 \\
\hline Damoh (MP) & 22.1 & 3.0 & 4.4 & 23.0 & 3.2 & 4.5 & 18.4 & 2.3 & 3.8 \\
\hline Satna (MP) & 21.9 & 2.9 & 4.4 & 22.8 & 3.1 & 4.6 & 18.7 & 2.3 & 3.7 \\
\hline Rewa (MP) & 21.8 & 2.9 & 4.4 & 22.5 & 3.1 & 4.6 & 18.2 & 2.2 & 3.6 \\
\hline Umaria (MP) & 23.2 & 3.0 & 4.5 & 24.2 & 3.2 & 4.7 & 18.1 & 2.1 & 3.5 \\
\hline Neemuch (MP) & 19.3 & 2.4 & 3.5 & 20.0 & 2.5 & 3.5 & 17.6 & 2.1 & 3.3 \\
\hline Mandsaur (MP) & 19.7 & 2.5 & 3.5 & 20.3 & 2.6 & 3.6 & 17.5 & 2.1 & 3.2 \\
\hline Ratlam (MP) & 21.8 & 2.8 & 4.1 & 23.7 & 3.2 & 4.5 & 17.5 & 2.1 & 3.3 \\
\hline Ujjain (MP) & 20.0 & 2.5 & 3.7 & 21.7 & 2.9 & 4.0 & 17.2 & 2.1 & 3.2 \\
\hline Shajapur (MP) & 21.4 & 2.9 & 4.0 & 21.8 & 3.0 & 4.1 & 19.7 & 2.5 & 3.7 \\
\hline Dewas (MP) & 21.4 & 2.8 & 4.0 & 22.4 & 3.0 & 4.3 & 18.8 & 2.3 & 3.5 \\
\hline Dhar (MP) & 23.9 & 3.1 & 4.6 & 24.6 & 3.3 & 4.8 & 20.9 & 2.6 & 3.9 \\
\hline Indore (MP) & 18.5 & 2.2 & 3.4 & 21.2 & 2.7 & 3.9 & 17.6 & 2.1 & 3.2 \\
\hline Khargone (West Nimar) (MP) & 23.4 & 3.1 & 4.7 & 24.2 & 3.3 & 4.8 & 18.8 & 2.3 & 3.7 \\
\hline Barwani (MP) & 27.6 & 3.9 & 5.6 & 28.9 & 4.2 & 5.9 & 20.1 & 2.5 & 4.0 \\
\hline Rajgarh (MP) & 22.1 & 3.0 & 4.2 & 22.5 & 3.1 & 4.3 & 20.7 & 2.6 & 4.1 \\
\hline Vidisha (MP) & 23.9 & 3.4 & 5.1 & 24.9 & 3.8 & 5.4 & 20.4 & 2.6 & 4.1 \\
\hline Bhopal (MP) & 18.6 & 2.3 & 3.6 & 23.7 & 3.3 & 5.0 & 17.4 & 2.1 & 3.3 \\
\hline
\end{tabular}




\begin{tabular}{|c|c|c|c|c|c|c|c|c|c|}
\hline Sehore (MP) & 22.0 & 3.0 & 4.4 & 22.7 & 3.2 & 4.6 & 19.1 & 2.4 & 3.8 \\
\hline Raisen (MP) & 22.8 & 3.2 & 4.7 & 23.3 & 3.3 & 4.9 & 20.9 & 2.7 & 4.2 \\
\hline Betul (MP) & 19.6 & 2.4 & 4.0 & 20.6 & 2.6 & 4.2 & 15.6 & 1.7 & 3.0 \\
\hline Harda (MP) & 21.4 & 2.8 & 4.2 & 22.3 & 3.0 & 4.4 & 18.1 & 2.2 & 3.6 \\
\hline Hoshangabad (MP) & 19.4 & 2.5 & 3.9 & 20.7 & 2.8 & 4.2 & 16.4 & 1.9 & 3.3 \\
\hline Katni (MP) & 21.9 & 2.8 & 4.2 & 23.0 & 3.1 & 4.5 & 17.5 & 2.1 & 3.4 \\
\hline Jabalpur (MP) & 17.5 & 2.1 & 3.4 & 20.1 & 2.6 & 3.8 & 15.6 & 1.8 & 3.0 \\
\hline Narsimhapur (MP) & 19.0 & 2.4 & 3.6 & 19.5 & 2.5 & 3.7 & 16.5 & 2.0 & 3.2 \\
\hline Dindori (MP) & 22.4 & 2.8 & 4.2 & 22.6 & 2.8 & 4.2 & 18.4 & 2.1 & 3.5 \\
\hline Mandla (MP) & 20.5 & 2.5 & 3.9 & 21.2 & 2.6 & 4.0 & 15.9 & 1.8 & 3.2 \\
\hline Chhindwara (MP) & 19.1 & 2.3 & 3.9 & 20.1 & 2.5 & 4.1 & 15.8 & 1.8 & 3.2 \\
\hline Seoni (MP) & 19.3 & 2.4 & 3.8 & 19.8 & 2.4 & 3.9 & 16.0 & 1.8 & 3.2 \\
\hline Balaghat (MP) & 18.5 & 2.2 & 3.6 & 18.9 & 2.2 & 3.7 & 15.9 & 1.8 & 3.1 \\
\hline Guna (MP) & 24.4 & 3.4 & 4.9 & 25.5 & 3.7 & 5.1 & 21.1 & 2.7 & 4.1 \\
\hline Ashoknagar (MP) & 24.1 & 3.4 & 4.8 & 24.8 & 3.6 & 5.0 & 21.1 & 2.7 & 4.2 \\
\hline Shahdol (MP) & 21.6 & 2.7 & 4.0 & 22.8 & 2.9 & 4.2 & 17.1 & 2.0 & 3.3 \\
\hline Anuppur (MP) & 20.4 & 2.5 & 3.8 & 21.6 & 2.7 & 4.0 & 17.3 & 2.0 & 3.3 \\
\hline Sidhi (MP) & 24.9 & 3.4 & 5.0 & 25.3 & 3.5 & 5.1 & 20.7 & 2.6 & 4.1 \\
\hline Singrauli (MP) & 25.5 & 3.6 & 5.2 & 26.8 & 3.9 & 5.5 & 20.1 & 2.5 & 3.9 \\
\hline Jhabua (MP) & 29.7 & 4.3 & 6.1 & 30.6 & 4.5 & 6.3 & 20.8 & 2.5 & 4.0 \\
\hline Alirajpur (MP) & 29.5 & 4.2 & 6.5 & 30.2 & 4.4 & 6.7 & 21.3 & 2.6 & 4.3 \\
\hline Khandwa (East Nimar) (MP) & 23.0 & 3.1 & 4.7 & 24.1 & 3.3 & 4.9 & 18.6 & 2.3 & 3.8 \\
\hline Burhanpur (MP) & 23.2 & 3.1 & 4.7 & 25.2 & 3.5 & 5.1 & 19.2 & 2.4 & 3.9 \\
\hline GUJARAT (GJ) & 18.7 & 2.3 & 3.5 & 20.2 & 2.6 & 3.8 & 16.7 & 2.0 & 3.0 \\
\hline Kachchh (GJ) & 21.7 & 2.8 & 4.3 & 23.2 & 3.1 & 4.7 & 18.9 & 2.3 & 3.5 \\
\hline Banas Kantha (GJ) & 23.8 & 3.1 & 4.6 & 24.5 & 3.3 & 4.8 & 19.4 & 2.3 & 3.6 \\
\hline Patan (GJ) & 20.2 & 2.6 & 3.8 & 21.1 & 2.7 & 4.0 & 16.8 & 2.0 & 3.1 \\
\hline Mahesana (GJ) & 17.2 & 2.1 & 3.1 & 17.8 & 2.2 & 3.2 & 15.6 & 1.8 & 2.7 \\
\hline Sabar Kantha (GJ) & 20.3 & 2.6 & 3.8 & 20.8 & 2.6 & 3.9 & 17.7 & 2.1 & 3.2 \\
\hline Gandhinagar (GJ) & 17.4 & 2.1 & 3.0 & 18.3 & 2.2 & 3.2 & 16.3 & 1.9 & 2.8 \\
\hline Ahmadabad (GJ) & 16.9 & 2.0 & 3.0 & 20.3 & 2.6 & 3.7 & 16.2 & 1.9 & 2.9 \\
\hline Surendranagar (GJ) & 20.0 & 2.6 & 3.9 & 21.3 & 2.8 & 4.2 & 16.6 & 2.0 & 3.1 \\
\hline Rajkot (GJ) & 16.8 & 2.0 & 3.0 & 17.6 & 2.2 & 3.3 & 16.2 & 1.9 & 2.8 \\
\hline Jamnagar (GJ) & 18.0 & 2.2 & 3.5 & 18.8 & 2.4 & 3.7 & 17.0 & 2.0 & 3.1 \\
\hline Porbandar (GJ) & 16.9 & 2.1 & 3.2 & 17.9 & 2.2 & 3.4 & 15.9 & 1.9 & 3.0 \\
\hline \multicolumn{10}{|c|}{ Appendix Table 1: Indirect estimates of CBR,TFR \& TMFR-Total, Rural and Urban areas-India/States/UTs/Districts-2007 } \\
\hline & \multicolumn{3}{|c|}{ TOTAL - 2007} & \multicolumn{3}{|c|}{ RURAL - 2007} & \multicolumn{3}{|c|}{ URBAN - 2007} \\
\hline India/States/Uts/Districts & CBR & TFR & TMFR & CBR & TFR & TMFR & CBR & TFR & TMFR \\
\hline Junagadh (GJ) & 17.1 & 2.1 & 3.4 & 17.6 & 2.2 & 3.5 & 16.2 & 1.9 & 3.1 \\
\hline Amreli (GJ) & 17.0 & 2.1 & 3.4 & 17.3 & 2.2 & 3.5 & 16.2 & 1.9 & 3.1 \\
\hline Bhavnagar (GJ) & 19.5 & 2.4 & 3.9 & 20.9 & 2.7 & 4.3 & 17.5 & 2.1 & 3.3 \\
\hline Anand (GJ) & 17.6 & 2.2 & 3.2 & 18.3 & 2.3 & 3.3 & 16.1 & 1.9 & 2.9 \\
\hline Kheda (GJ) & 18.4 & 2.3 & 3.3 & 18.9 & 2.4 & 3.4 & 16.5 & 2.0 & 3.1 \\
\hline Panch Mahals (GJ) & 21.7 & 2.8 & 4.2 & 22.2 & 2.9 & 4.3 & 18.6 & 2.2 & 3.4 \\
\hline Dohad (GJ) & 27.5 & 3.8 & 5.6 & 28.1 & 3.9 & 5.8 & 20.6 & 2.5 & 3.9 \\
\hline Vadodara (GJ) & 17.4 & 2.1 & 3.1 & 19.9 & 2.5 & 3.6 & 15.0 & 1.7 & 2.6 \\
\hline Narmada (GJ) & 19.4 & 2.4 & 3.7 & 19.9 & 2.5 & 3.8 & 14.8 & 1.7 & 2.8 \\
\hline Bharuch (GJ) & 17.1 & 2.1 & 3.2 & 17.6 & 2.2 & 3.4 & 16.2 & 1.9 & 2.9 \\
\hline The Dangs (GJ) & 26.1 & 3.4 & 5.1 & 27.2 & 3.7 & 5.2 & 17.1 & 2.0 & 3.8 \\
\hline Navsari (GJ) & 14.9 & 1.7 & 2.7 & 14.8 & 1.7 & 2.7 & 15.0 & 1.7 & 2.7 \\
\hline Valsad (GJ) & 18.4 & 2.3 & 3.4 & 19.5 & 2.4 & 3.6 & 16.7 & 2.0 & 3.1 \\
\hline Surat (GJ) & 17.3 & 2.2 & 3.2 & 16.6 & 2.0 & 3.1 & 17.5 & 2.2 & 3.2 \\
\hline Tapi (GJ) & 16.3 & 1.9 & 2.9 & 16.3 & 1.9 & 2.9 & 16.4 & 1.9 & 3.0 \\
\hline DAMAN \& DIU (DD) & 15.5 & 2.3 & 3.5 & 18.1 & 2.3 & 3.9 & 14.6 & 2.3 & 3.4 \\
\hline Diu (DD) & 18.7 & 2.2 & 4.1 & 21.5 & 2.7 & 5.0 & 15.5 & 1.7 & 3.1 \\
\hline Daman (DD) & 14.6 & 2.3 & 3.3 & 15.2 & 2.0 & 3.0 & 14.5 & 2.4 & 3.4 \\
\hline DADRA \& NAGAR HAVELI (DN) & 21.2 & 2.9 & 4.3 & 22.7 & 3.1 & 4.8 & 19.4 & 2.7 & 3.7 \\
\hline Dadra \& Nagar Haveli (DN) & 21.2 & 2.9 & 4.3 & 22.7 & 3.1 & 4.8 & 19.4 & 2.7 & 3.7 \\
\hline MAHARASHTRA (MH) & 17.1 & 2.1 & 3.2 & 18.0 & 2.3 & 3.4 & 16.0 & 1.9 & 3.0 \\
\hline Nandurbar (MH) & 21.6 & 2.8 & 4.3 & 22.4 & 2.9 & 4.4 & 17.7 & 2.2 & 3.6 \\
\hline Dhule (MH) & 19.5 & 2.5 & 3.8 & 20.4 & 2.7 & 4.0 & 17.2 & 2.1 & 3.4 \\
\hline Jalgaon (MH) & 18.1 & 2.3 & 3.5 & 18.7 & 2.4 & 3.6 & 16.9 & 2.0 & 3.2 \\
\hline Buldana (MH) & 18.3 & 2.3 & 3.5 & 18.4 & 2.4 & 3.5 & 17.6 & 2.1 & 3.4 \\
\hline Akola (MH) & 16.8 & 2.1 & 3.3 & 17.0 & 2.1 & 3.3 & 16.5 & 1.9 & 3.2 \\
\hline Washim (MH) & 18.3 & 2.4 & 3.6 & 18.4 & 2.4 & 3.6 & 18.3 & 2.3 & 3.6 \\
\hline Amravati (MH) & 15.7 & 1.9 & 3.1 & 16.1 & 2.0 & 3.2 & 15.0 & 1.7 & 2.9 \\
\hline Wardha (MH) & 14.4 & 1.7 & 2.8 & 14.6 & 1.8 & 2.8 & 14.0 & 1.6 & 2.6 \\
\hline Nagpur (MH) & 15.3 & 1.8 & 2.9 & 15.9 & 1.9 & 3.1 & 15.1 & 1.7 & 2.8 \\
\hline Bhandara (MH) & 15.3 & 1.8 & 3.0 & 15.6 & 1.9 & 3.0 & 14.3 & 1.6 & 2.8 \\
\hline Gondiya (MH) & 15.7 & 1.8 & 3.0 & 15.9 & 1.9 & 3.0 & 14.8 & 1.7 & 2.8 \\
\hline
\end{tabular}




\begin{tabular}{|c|c|c|c|c|c|c|c|c|c|}
\hline Gadchiroli (MH) & 16.7 & 2.0 & 3.2 & 16.9 & 2.0 & 3.2 & 14.8 & 1.6 & 2.8 \\
\hline Chandrapur (MH) & 15.2 & 1.8 & 2.8 & 15.6 & 1.9 & 2.9 & 14.5 & 1.6 & 2.6 \\
\hline Yavatmal (MH) & 17.2 & 2.1 & 3.3 & 17.5 & 2.2 & 3.4 & 15.8 & 1.8 & 3.0 \\
\hline Nanded $(\mathrm{MH})$ & 19.8 & 2.6 & 3.9 & 20.1 & 2.7 & 3.9 & 19.0 & 2.3 & 3.7 \\
\hline Hingoli (MH) & 20.2 & 2.7 & 3.9 & 20.3 & 2.7 & 3.9 & 19.7 & 2.5 & 3.9 \\
\hline Parbhani (MH) & 20.0 & 2.7 & 3.9 & 20.2 & 2.7 & 3.9 & 19.7 & 2.5 & 3.9 \\
\hline Jalna (MH) & 20.7 & 2.8 & 4.1 & 20.9 & 2.9 & 4.1 & 19.9 & 2.5 & 3.8 \\
\hline Aurangabad (MH) & 20.4 & 2.6 & 3.8 & 20.8 & 2.8 & 4.0 & 19.8 & 2.4 & 3.6 \\
\hline Nashik $(\mathrm{MH})$ & 19.4 & 2.4 & 3.6 & 20.2 & 2.6 & 3.8 & 18.2 & 2.2 & 3.4 \\
\hline Thane $(\mathrm{MH})$ & 17.3 & 2.1 & 3.2 & 20.7 & 2.6 & 3.9 & 16.2 & 1.9 & 2.9 \\
\hline Mumbai Suburban (MH) & 14.4 & 1.7 & 2.8 & NA & NA & NA & 14.4 & 1.7 & 2.8 \\
\hline Mumbai (MH) & 12.9 & 1.6 & 2.7 & NA & NA & NA & 12.9 & 1.6 & 2.7 \\
\hline Raigarh (MH) & 16.5 & 1.9 & 3.0 & 16.5 & 2.0 & 3.1 & 16.5 & 1.9 & 2.8 \\
\hline Pune $(\mathrm{MH})$ & 16.5 & 1.9 & 2.9 & 16.9 & 2.1 & 3.1 & 16.1 & 1.9 & 2.9 \\
\hline Ahmadnagar (MH) & 17.6 & 2.2 & 3.2 & 17.8 & 2.3 & 3.3 & 16.7 & 2.0 & 3.0 \\
\hline $\operatorname{Bid}(\mathrm{MH})$ & 19.5 & 2.6 & 3.8 & 19.5 & 2.7 & 3.8 & 19.4 & 2.4 & 3.7 \\
\hline Latur (MH) & 18.8 & 2.5 & 3.8 & 18.8 & 2.6 & 3.8 & 18.7 & 2.3 & 3.6 \\
\hline Osmanabad (MH) & 17.8 & 2.4 & 3.6 & 17.7 & 2.4 & 3.6 & 18.4 & 2.3 & 3.6 \\
\hline Solapur (MH) & 17.9 & 2.2 & 3.4 & 18.4 & 2.4 & 3.5 & 16.8 & 2.0 & 3.2 \\
\hline Satara $(\mathrm{MH})$ & 15.4 & 1.9 & 2.8 & 15.4 & 1.9 & 2.9 & 15.4 & 1.8 & 2.8 \\
\hline Ratnagiri (MH)) & 14.5 & 1.7 & 2.8 & 14.5 & 1.7 & 2.8 & 14.9 & 1.7 & 2.7 \\
\hline Sindhudurg (MH) & 12.7 & 1.5 & 2.5 & 12.6 & 1.5 & 2.5 & 13.3 & 1.5 & 2.5 \\
\hline Kolhapur (MH) & 15.3 & 1.8 & 2.7 & 15.3 & 1.9 & 2.8 & 15.2 & 1.7 & 2.7 \\
\hline Sangli (MH) & 15.8 & 1.9 & 2.9 & 15.9 & 2.0 & 2.9 & 15.5 & 1.8 & 2.8 \\
\hline \multicolumn{10}{|l|}{ ANDHRA PRADESH } \\
\hline$(O L D)(A P+T S)$ & 16.1 & 1.9 & 2.9 & 16.3 & 2.0 & 3.0 & 15.6 & 1.7 & 2.8 \\
\hline TELANGANA STATE (TS) & 16.5 & 2.0 & 3.0 & 16.6 & 2.0 & 3.1 & 16.4 & 1.9 & 3.0 \\
\hline Adilabad (TS) & 17.0 & 2.1 & 3.3 & 17.8 & 2.2 & 3.4 & 14.9 & 1.7 & 2.8 \\
\hline Nizamabad (TS) & 16.6 & 2.0 & 3.1 & 16.4 & 2.0 & 3.0 & 17.1 & 2.0 & 3.3 \\
\hline Karimnagar (TS) & 13.6 & 1.6 & 2.5 & 13.3 & 1.6 & 2.5 & 14.4 & 1.6 & 2.6 \\
\hline Medak (TS) & 17.8 & 2.2 & 3.3 & 17.9 & 2.2 & 3.4 & 17.5 & 2.0 & 3.1 \\
\hline Hyderabad (TS) & 17.3 & 2.0 & 3.3 & NA & NA & NA & 17.3 & 2.0 & 3.3 \\
\hline Rangareddy (TS) & 17.2 & 2.0 & 3.0 & 18.0 & 2.3 & 3.5 & 16.8 & 1.9 & 2.9 \\
\hline Mahbubnagar (TS) & 19.3 & 2.4 & 3.6 & 19.7 & 2.5 & 3.7 & 17.3 & 2.0 & 3.3 \\
\hline Nalgonda (TS) & 15.9 & 1.9 & 2.9 & 16.0 & 2.0 & 2.9 & 15.4 & 1.7 & 2.8 \\
\hline Warangal (TS) & 14.8 & 1.8 & 2.7 & 15.0 & 1.8 & 2.8 & 14.3 & 1.6 & 2.6 \\
\hline Khammam (TS) & 15.2 & 1.7 & 2.7 & 15.4 & 1.8 & 2.8 & 14.6 & 1.6 & 2.5 \\
\hline ANDHRA PRADESH (New) (AP) & 15.7 & 1.8 & 2.8 & 16.1 & 1.9 & 2.9 & 14.9 & 1.6 & 2.6 \\
\hline Srikakulam (AP) & 15.7 & 1.8 & 2.9 & 15.8 & 1.9 & 2.9 & 14.7 & 1.6 & 2.6 \\
\hline Vizianagaram (AP) & 15.5 & 1.8 & 2.8 & 15.8 & 1.9 & 2.9 & 14.2 & 1.6 & 2.5 \\
\hline \multicolumn{10}{|c|}{ Appendix Table 1: Indirect estimates of CBR,TFR \& TMFR -Total, Rural and Urban areas-India/States/UTs/Districts-2007 } \\
\hline & \multicolumn{3}{|c|}{ TOTAL - 2007} & \multicolumn{3}{|c|}{ RURAL - 2007} & \multicolumn{3}{|c|}{ URBAN - 2007} \\
\hline India/States/Uts/Districts & CBR & TFR & TMFR & CBR & TFR & TMFR & CBR & TFR & TMFR \\
\hline Visakhapatnam (AP) & 15.6 & 1.8 & 2.7 & 17.1 & 2.0 & 3.1 & 14.0 & 1.5 & 2.4 \\
\hline East Godavari (AP) & 15.1 & 1.7 & 2.7 & 15.4 & 1.8 & 2.7 & 14.4 & 1.5 & 2.5 \\
\hline West Godavari (AP) & 14.6 & 1.7 & 2.6 & 14.8 & 1.7 & 2.6 & 13.7 & 1.5 & 2.4 \\
\hline Krishna (AP) & 14.3 & 1.6 & 2.5 & 14.4 & 1.7 & 2.6 & 14.1 & 1.5 & 2.5 \\
\hline Guntur (AP) & 15.1 & 1.7 & 2.6 & 15.3 & 1.8 & 2.7 & 14.7 & 1.6 & 2.6 \\
\hline Prakasam (AP) & 16.6 & 2.0 & 2.9 & 17.0 & 2.1 & 3.0 & 15.1 & 1.7 & 2.6 \\
\hline Sri Potti Sriramulu Nellore (AP) & 15.3 & 1.7 & 2.7 & 15.8 & 1.8 & 2.8 & 13.9 & 1.5 & 2.5 \\
\hline Y.S.R. (AP) & 17.0 & 2.0 & 3.1 & 17.2 & 2.1 & 3.1 & 16.7 & 1.9 & 3.0 \\
\hline Kurnool (AP) & 18.6 & 2.2 & 3.4 & 19.1 & 2.4 & 3.5 & 17.4 & 2.0 & 3.2 \\
\hline Anantapur (AP) & 16.2 & 1.9 & 2.9 & 16.4 & 2.0 & 3.0 & 15.9 & 1.8 & 2.9 \\
\hline Chittoor (AP) & 15.7 & 1.9 & 2.9 & 16.0 & 1.9 & 2.9 & 14.9 & 1.7 & 2.7 \\
\hline KARNATAKA (KA) & 16.8 & 2.0 & 3.1 & 17.3 & 2.1 & 3.3 & 16.1 & 1.8 & 2.9 \\
\hline Belgaum (KA) & 18.7 & 2.3 & 3.5 & 19.4 & 2.5 & 3.7 & 16.6 & 1.9 & 3.0 \\
\hline Bagalkot (KA) & 20.6 & 2.6 & 4.0 & 21.4 & 2.7 & 4.1 & 18.9 & 2.2 & 3.6 \\
\hline Bijapur (KA) & 20.9 & 2.7 & 4.1 & 21.4 & 2.8 & 4.2 & 19.3 & 2.3 & 3.7 \\
\hline Bidar (KA) & 19.3 & 2.5 & 4.0 & 19.3 & 2.6 & 4.1 & 19.5 & 2.4 & 3.9 \\
\hline Raichur (KA) & 21.1 & 2.6 & 4.1 & 21.8 & 2.7 & 4.2 & 19.1 & 2.2 & 3.7 \\
\hline Koppal (KA) & 20.8 & 2.6 & 4.2 & 21.1 & 2.7 & 4.2 & 19.2 & 2.3 & 3.7 \\
\hline Gadag (KA) & 17.7 & 2.1 & 3.4 & 18.0 & 2.2 & 3.5 & 17.1 & 2.0 & 3.3 \\
\hline Dharwad (KA) & 17.0 & 2.0 & 3.2 & 17.9 & 2.2 & 3.4 & 16.4 & 1.9 & 3.1 \\
\hline Uttara Kannada (KA) & 15.1 & 1.8 & 2.9 & 15.2 & 1.8 & 2.9 & 15.0 & 1.7 & 2.9 \\
\hline Haveri (KA) & 17.5 & 2.1 & 3.4 & 17.4 & 2.1 & 3.4 & 17.7 & 2.1 & 3.5 \\
\hline Bellary (KA) & 20.0 & 2.4 & 3.8 & 20.7 & 2.6 & 4.0 & 18.8 & 2.2 & 3.4 \\
\hline Chitradurga (KA) & 16.0 & 1.9 & 2.9 & 16.0 & 1.9 & 2.9 & 15.6 & 1.7 & 2.8 \\
\hline Davanagere (KA) & 16.2 & 1.9 & 3.0 & 16.2 & 1.9 & 3.0 & 16.3 & 1.8 & 2.9 \\
\hline Shimoga $(K A)$ & 15.3 & 1.7 & 2.8 & 15.5 & 1.8 & 2.8 & 14.8 & 1.6 & 2.7 \\
\hline Udupi (KA) & 12.7 & 1.4 & 2.4 & 12.8 & 1.4 & 2.4 & 12.5 & 1.4 & 2.3 \\
\hline
\end{tabular}




\begin{tabular}{|c|c|c|c|c|c|c|c|c|c|}
\hline Chikmagalur (KA) & 13.6 & 1.5 & 2.4 & 13.5 & 1.5 & 2.4 & 14.3 & 1.5 & 2.6 \\
\hline Tumkur (KA) & 14.4 & 1.7 & 2.6 & 14.2 & 1.7 & 2.6 & 15.2 & 1.7 & 2.7 \\
\hline Bangalore (KA) & 15.4 & 1.7 & 2.6 & 16.1 & 1.9 & 2.8 & 15.3 & 1.7 & 2.6 \\
\hline Mandya (KA) & 14.0 & 1.6 & 2.5 & 13.7 & 1.6 & 2.4 & 15.0 & 1.6 & 2.6 \\
\hline Hassan (KA) & 13.7 & 1.6 & 2.4 & 13.4 & 1.5 & 2.3 & 14.8 & 1.6 & 2.6 \\
\hline Dakshina Kannada (KA) & 14.5 & 1.6 & 2.8 & 14.8 & 1.6 & 2.9 & 14.2 & 1.5 & 2.7 \\
\hline Kodagu (KA) & 14.7 & 1.7 & 2.7 & 14.7 & 1.7 & 2.7 & 14.6 & 1.6 & 2.7 \\
\hline Mysore (KA) & 14.8 & 1.7 & 2.6 & 15.2 & 1.8 & 2.7 & 14.3 & 1.5 & 2.5 \\
\hline Chamarajanagar (KA) & 14.3 & 1.6 & 2.5 & 14.2 & 1.6 & 2.5 & 15.0 & 1.6 & 2.7 \\
\hline Gulbarga (KA) & 20.6 & 2.6 & 4.2 & 21.2 & 2.8 & 4.4 & 19.3 & 2.3 & 3.8 \\
\hline Yadgir (KA) & 23.2 & 3.0 & 4.8 & 23.7 & 3.2 & 4.9 & 21.1 & 2.6 & 4.4 \\
\hline Kolar (KA) & 16.2 & 1.9 & 3.0 & 16.0 & 1.9 & 3.0 & 16.5 & 1.9 & 3.1 \\
\hline Chikkaballapura (KA) & 15.4 & 1.8 & 2.7 & 15.0 & 1.8 & 2.7 & 16.8 & 1.9 & 3.0 \\
\hline Bangalore Rural (KA) & 15.4 & 1.8 & 2.8 & 15.2 & 1.8 & 2.8 & 15.9 & 1.8 & 2.8 \\
\hline Ramanagara $(\mathrm{KA})$ & 14.4 & 1.7 & 2.7 & 13.7 & 1.6 & 2.6 & 16.5 & 1.9 & 3.1 \\
\hline$G O A(G A)$ & 14.2 & 1.6 & 2.7 & 14.0 & 1.6 & 2.7 & 14.3 & 1.7 & 2.8 \\
\hline North Goa (GA) & 13.6 & 1.6 & 2.6 & 13.5 & 1.6 & 2.6 & 13.7 & 1.6 & 2.6 \\
\hline South Goa (GA) & 15.0 & 1.7 & 2.9 & 14.7 & 1.7 & 2.9 & 15.1 & 1.7 & 2.9 \\
\hline LAKSHADWEEP (LD) & 16.2 & 1.9 & 3.2 & 18.5 & 2.3 & 3.7 & 15.6 & 1.8 & 3.1 \\
\hline Lakshadweep (LD) & 16.2 & 1.9 & 3.2 & 18.5 & 2.3 & 3.7 & 15.6 & 1.8 & 3.1 \\
\hline $\operatorname{KERALA}(K L)$ & 15.0 & 1.7 & 2.6 & 15.1 & 1.7 & 2.7 & 14.9 & 1.7 & 2.6 \\
\hline Kasaragod (KL) & 16.9 & 1.9 & 3.1 & 16.4 & 1.8 & 3.0 & 17.6 & 1.9 & 3.1 \\
\hline Kannur (KL) & 15.3 & 1.7 & 2.6 & 15.2 & 1.7 & 2.7 & 15.4 & 1.7 & 2.6 \\
\hline Wayanad (KL) & 16.5 & 1.9 & 2.9 & 16.5 & 1.9 & 2.9 & 16.5 & 1.8 & 2.8 \\
\hline Kozhikode (KL) & 15.7 & 1.7 & 2.6 & 16.2 & 1.8 & 2.7 & 15.4 & 1.7 & 2.6 \\
\hline Malappuram (KL) & 19.9 & 2.2 & 3.4 & 19.9 & 2.2 & 3.4 & 19.9 & 2.2 & 3.4 \\
\hline Palakkad (KL) & 15.4 & 1.8 & 2.8 & 15.6 & 1.8 & 2.8 & 15.0 & 1.7 & 2.7 \\
\hline Thrissur (KL) & 14.0 & 1.6 & 2.5 & 14.2 & 1.6 & 2.5 & 14.0 & 1.6 & 2.4 \\
\hline Ernakulam (KL) & 13.4 & 1.6 & 2.4 & 13.0 & 1.6 & 2.4 & 13.6 & 1.6 & 2.4 \\
\hline Idukki (KL) & 14.0 & 1.6 & 2.5 & 14.0 & 1.6 & 2.5 & 14.7 & 1.7 & 2.7 \\
\hline Kottayam (KL) & 13.1 & 1.6 & 2.5 & 13.1 & 1.6 & 2.5 & 13.0 & 1.5 & 2.5 \\
\hline Alappuzha (KL) & 13.2 & 1.5 & 2.3 & 13.3 & 1.5 & 2.3 & 13.1 & 1.5 & 2.3 \\
\hline Pathanamthitta (KL) & 12.0 & 1.4 & 2.2 & 12.0 & 1.4 & 2.2 & 12.1 & 1.4 & 2.2 \\
\hline Kollam (KL) & 14.1 & 1.6 & 2.4 & 13.9 & 1.5 & 2.4 & 14.3 & 1.6 & 2.4 \\
\hline Thiruvananthapuram (KL) & 13.4 & 1.5 & 2.3 & 14.0 & 1.5 & 2.4 & 13.0 & 1.5 & 2.3 \\
\hline TAMIL NADU $(T N)$ & 15.0 & 1.7 & 2.7 & 15.4 & 1.8 & 2.8 & 14.5 & 1.6 & 2.5 \\
\hline Thiruvallur (TN) & 15.5 & 1.7 & 2.6 & 15.7 & 1.8 & 2.8 & 15.3 & 1.7 & 2.5 \\
\hline Chennai (TN) & 14.0 & 1.5 & 2.4 & NA & NA & NA & 14.0 & 1.5 & 2.4 \\
\hline Kancheepuram (TN) & 15.2 & 1.7 & 2.7 & 15.6 & 1.7 & 2.8 & 15.0 & 1.6 & 2.5 \\
\hline Vellore (TN) & 16.0 & 1.8 & 2.9 & 16.2 & 1.9 & 3.0 & 15.6 & 1.7 & 2.9 \\
\hline Tiruvannamalai (TN) & 16.1 & 1.9 & 2.9 & 16.3 & 1.9 & 3.0 & 15.3 & 1.7 & 2.8 \\
\hline Viluppuram (TN) & 17.1 & 2.0 & 3.1 & 17.3 & 2.0 & 3.2 & 15.9 & 1.8 & 2.8 \\
\hline Salem (TN) & 14.7 & 1.7 & 2.6 & 15.1 & 1.8 & 2.7 & 14.3 & 1.6 & 2.5 \\
\hline Namakkal (TN) & 13.0 & 1.5 & 2.3 & 12.8 & 1.5 & 2.4 & 13.1 & 1.5 & 2.3 \\
\hline Erode (TN) & 12.7 & 1.4 & 2.2 & 12.5 & 1.4 & 2.2 & 13.0 & 1.4 & 2.2 \\
\hline \multicolumn{10}{|c|}{ Appendix Table 1: Indirect estimates of CBR,TFR \&TMFR -Total, Rural and Urban areas-India/States/UTs/Districts-2007 } \\
\hline & \multicolumn{3}{|c|}{ TOTAL - 2007} & \multicolumn{3}{|c|}{ RURAL - 2007} & \multicolumn{3}{|c|}{ URBAN - 2007} \\
\hline India/States/Uts/Districts & CBR & TFR & TMFR & CBR & TFR & TMFR & CBR & TFR & TMFR \\
\hline The Nilgiris (TN) & 13.6 & 1.5 & 2.4 & 13.2 & 1.4 & 2.3 & 14.0 & 1.5 & 2.5 \\
\hline Dindigul (TN) & 14.7 & 1.7 & 2.6 & 14.8 & 1.7 & 2.6 & 14.4 & 1.6 & 2.5 \\
\hline Karur (TN) & 14.2 & 1.6 & 2.5 & 14.3 & 1.7 & 2.6 & 14.0 & 1.5 & 2.4 \\
\hline Tiruchirappalli (TN) & 14.7 & 1.7 & 2.7 & 15.5 & 1.8 & 2.9 & 13.9 & 1.5 & 2.5 \\
\hline Perambalur (TN) & 15.8 & 1.8 & 2.8 & 15.8 & 1.8 & 2.8 & 15.7 & 1.7 & 2.7 \\
\hline Ariyalur (TN) & 16.1 & 1.9 & 2.9 & 16.2 & 1.9 & 3.0 & 15.4 & 1.7 & 2.8 \\
\hline Cuddalore (TN) & 15.8 & 1.8 & 2.8 & 16.5 & 1.9 & 3.0 & 14.6 & 1.6 & 2.6 \\
\hline Nagapattinam (TN) & 15.1 & 1.7 & 2.8 & 15.1 & 1.7 & 2.8 & 15.0 & 1.7 & 2.7 \\
\hline Thiruvarur (TN) & 14.3 & 1.6 & 2.6 & 14.2 & 1.6 & 2.6 & 14.4 & 1.6 & 2.5 \\
\hline Thanjavur (TN) & 14.7 & 1.6 & 2.7 & 15.0 & 1.7 & 2.8 & 14.2 & 1.5 & 2.5 \\
\hline Pudukkottai (TN) & 16.4 & 1.9 & 3.0 & 16.6 & 1.9 & 3.1 & 15.7 & 1.7 & 2.8 \\
\hline Sivaganga (TN) & 15.2 & 1.7 & 2.7 & 15.2 & 1.8 & 2.8 & 15.0 & 1.7 & 2.6 \\
\hline Madurai (TN) & 15.1 & 1.7 & 2.7 & 15.9 & 1.8 & 2.9 & 14.7 & 1.6 & 2.5 \\
\hline Theni (TN) & 14.3 & 1.6 & 2.5 & 14.5 & 1.7 & 2.6 & 14.0 & 1.6 & 2.4 \\
\hline Virudhunagar (TN) & 15.1 & 1.7 & 2.7 & 15.6 & 1.8 & 2.9 & 14.6 & 1.6 & 2.5 \\
\hline Ramanathapuram (TN) & 15.4 & 1.8 & 2.7 & 15.2 & 1.8 & 2.7 & 15.8 & 1.8 & 2.8 \\
\hline Thoothukkudi (TN) & 15.5 & 1.8 & 3.0 & 15.5 & 1.8 & 3.1 & 15.6 & 1.8 & 2.8 \\
\hline Tirunelveli (TN) & 15.4 & 1.8 & 2.9 & 15.9 & 1.9 & 3.0 & 15.0 & 1.7 & 2.7 \\
\hline Kanniyakumari (TN) & 14.2 & 1.6 & 2.6 & 14.6 & 1.7 & 2.7 & 14.1 & 1.6 & 2.6 \\
\hline Dharmapuri (TN) & 16.3 & 1.9 & 2.9 & 16.5 & 2.0 & 2.9 & 15.2 & 1.7 & 2.7 \\
\hline Krishnagiri (TN) & 16.8 & 2.0 & 3.0 & 16.7 & 2.0 & 3.0 & 17.1 & 1.9 & 2.9 \\
\hline Coimbatore (TN) & 13.3 & 1.5 & 2.2 & 12.3 & 1.4 & 2.1 & 13.6 & 1.5 & 2.3 \\
\hline
\end{tabular}




\begin{tabular}{|c|c|c|c|c|c|c|c|c|c|}
\hline Tiruppur (TN) & 13.9 & 1.5 & 2.3 & 12.2 & 1.4 & 2.1 & 15.0 & 1.6 & 2.4 \\
\hline PUDUCHERRY (PY) & 15.4 & 1.7 & 2.8 & 16.3 & 1.8 & 3.0 & 15.0 & 1.6 & 2.7 \\
\hline Yanam (PY) & 16.8 & 1.9 & 3.1 & NA & NA & NA & 16.8 & 1.9 & 3.1 \\
\hline Puducherry (PY) & 15.2 & 1.7 & 2.8 & 16.4 & 1.8 & 3.0 & 14.7 & 1.6 & 2.6 \\
\hline Mahe (PY) & 15.5 & 1.7 & 2.6 & NA & NA & NA & 15.5 & 1.7 & 2.6 \\
\hline Karaikal (PY) & 16.0 & 1.8 & 3.0 & 16.2 & 1.8 & 3.1 & 15.8 & 1.8 & 2.9 \\
\hline \multicolumn{10}{|l|}{ ANDAMAN \& NICOBAR } \\
\hline ISLANDS (AN) & 15.6 & 1.8 & 2.9 & 16.0 & 1.9 & 3.0 & 14.8 & 1.7 & 2.6 \\
\hline Nicobars (AN) & 15.8 & 2.0 & 3.5 & 15.8 & 2.0 & 3.5 & NA & NA & NA \\
\hline North \& Middle Andaman (AN) & 16.5 & 1.9 & 3.0 & 16.6 & 2.0 & 3.1 & 13.3 & 1.4 & 2.4 \\
\hline South Andaman (AN) & 15.1 & 1.7 & 2.7 & 15.5 & 1.8 & 2.8 & 14.9 & 1.7 & 2.6 \\
\hline
\end{tabular}

